Article

\title{
Highly Efficient and Diastereoselective Synthesis of New Pyrazolylpyrrolizine and Pyrazolylpyrrolidine Derivates by a Three-Component Domino Process
}

\author{
Jairo Quiroga ${ }^{1}{ }^{1}$, Jaime Gálvez ${ }^{1}$, Rodrigo Abonia ${ }^{1}$, Braulio Insuasty ${ }^{1}$, Alejandro Ortíz ${ }^{1}$, \\ Justo Cobo $^{2}$ and Manuel Nogueras ${ }^{2}$
}

1 Heterocyclic Compounds Research Group, Department of Chemistry, Universidad del Valle, Cali 760032, Colombia; E-Mails: jaimegalvez.n@gmail.com (J.G.);

rodrigo.abonia@correounivalle.edu.co (R.A.); braulio.insuasty@correounivalle.edu.co (B.I.); alejandro.ortiz@correounivalle.edu.co (A.O.)

2 Department of Inorganic and Organic Chemistry, Universidad de Jaén, Jaén 23071, Spain;

E-Mails: jcobo@ujaen.es (J.C.); mmontiel@ujaen.es (M.N.)

* Author to whom correspondence should be addressed; E-Mail: jairo.quiroga@correounivalle.edu.co; Tel.: +57-2339-3240; Fax: +57-2339-2444.

Received: 6 February 2014; in revised form: 27 March 2014 / Accepted: 28 March 2014 /

Published: 4 April 2014

\begin{abstract}
Diastereoselective reactions between 4-formylpyrazoles, $N$-substituted maleimides and glycine derivates led to new series of pyrazolyldipyrrolo [3,4-a:3',4'-f]pyrrolizines and pyrazolylpyrrolo[3,4-c]pyrroles in good yields. The reactions proceeded by a domino process through azomethine ylides formed in situ via a 1,3-dipolar cycloaddition reaction.
\end{abstract}

Keywords: dipyrrolo [3,4-a:3',4'-f]pyrrolizines; pyrrolo[3,4-c]pirroles; 1,3-dipolar cycloadditions; multicomponent reactions; domino reactions

\section{Introduction}

Multicomponent reactions (MCRs) are one of the most powerful tools in organic synthesis allowing the formation of several bonds in one step to obtain products with high structural diversity and/or molecular complexity [1-3]. Furthermore, the development of fast, selective and environmentally friendly MCRs involving domino processes with step and atom economy are of great importance for medicinal and synthetic chemistry [4-6]. 
The dipolar cycloaddition reaction is a known and widely studied method in organic synthesis to obtain pyrrolidine derivatives from the reaction of azomethine ylides, generated in situ, and electrondeficient olefins [7-9]. These five-membered ring systems belong to an important class of aza-compounds with multiple applications, for example, in the development of bioactive molecules, organocatalysts, new materials and as scaffolds in total organic synthesis [10-13].

Some interesting fused pyrrolidine systems as cyclopiazonic acid, granulatimide and isogranulatimide (Figure 1) are natural alkaloids that displayed important activities as Chk1 inhibitors and antiplasmodial agents [14,15]. Pyrrolizines are alkaloids generally isolated from plants, insects, bacteria or fungi $[16,17]$ that have exhibited important antiproliferative activities $[18,19]$. In the same way, the pyrrolo[3,4-c]pyrroles have been widely applied in a variety of fields such as materials sciences, pharmaceuticals and agrochemistry [20-22].

Figure 1. Pyrrolidine systems present in natural alkaloids.

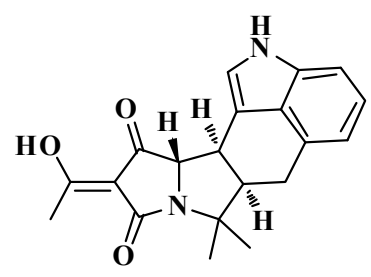

$\alpha-C y c l o p i a z o n i c ~ a c i d$

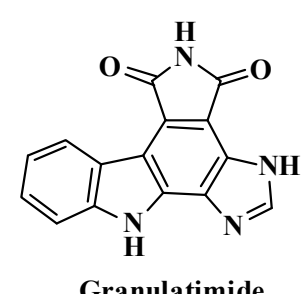

Granulatimide

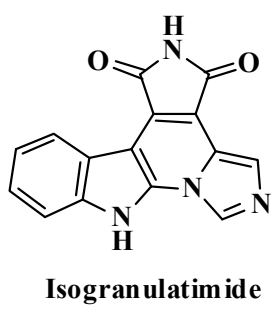

Pyrazole is another five-membered ring with many applications in chemistry, especially as pharmaceuticals, pesticides and lubricants [23-27]. In connection with our current studies on the development of new, selective, and environmentally friendly methodologies for the synthesis of fused heterocycles [28-31], herein we report a procedure for the preparation of the scarcely studied pyrazolylpyrrolizine derivates $\mathbf{4}$ and pyrazolylpyrrolidine derivatives $\mathbf{5}$ and $\mathbf{6}$ where three moieties of known importance (i.e., pyrazole, pyrrolidine, pyrrolizine) are incorporated into a single structure. The new compounds were obtained in good yields and high diastereoselectivity by a catalyst-free three-component domino reaction between formylpyrazoles $\mathbf{1}, \mathrm{N}$-arylmaleimides $\mathbf{2}$ and glycine-derived esters 3 (Scheme 1).

Scheme 1. Proposed synthesis of new pyrazolylpyrrolopyrrolizine derivates 4 and pyrazolylpyrrolopyrrolidine derivates 5 and $\mathbf{6}$.

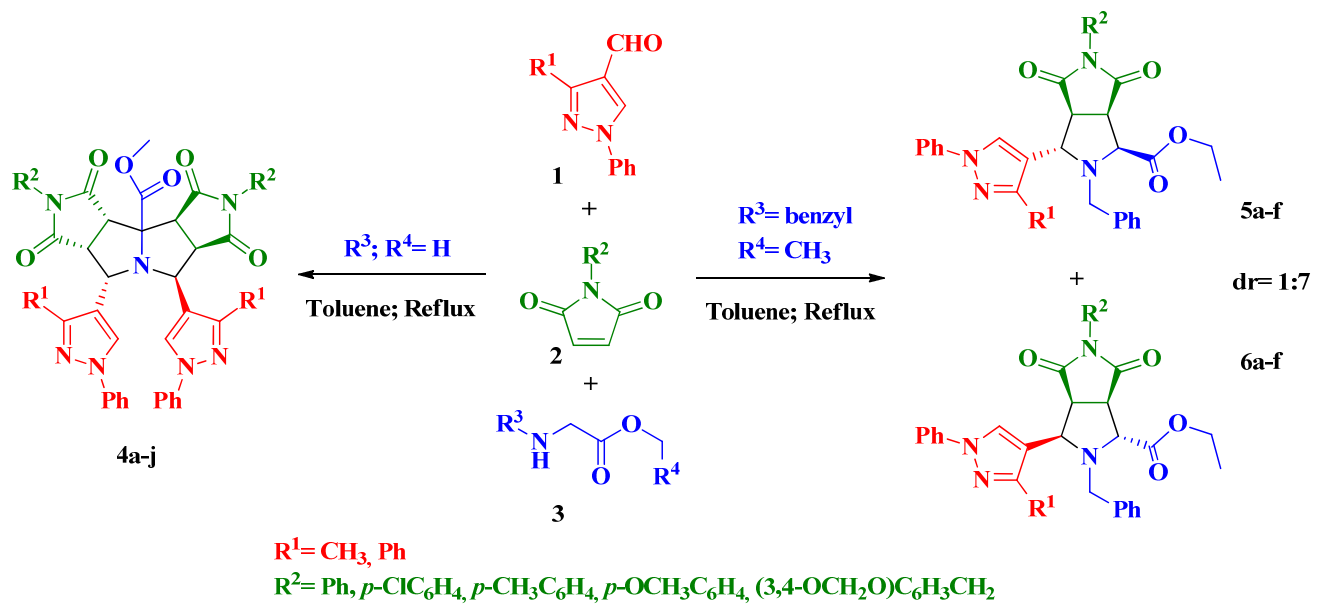




\section{Results and Discussion}

\subsection{Synthesis of Pyrazolyldipyrrolo[3,4-a:3',4'-f]Pyrrolizines from Glycine Methyl Ester}

To the best of our knowledge, not many pyrrolo[3,4-a:3',4'-f]pyrrolizine derivatives have been synthesized and few of them via 1,3-dipolar reactions [32-34]. Recently Zhang and coworkers reported the synthesis of pyrrolopyrrolizines from 2-furanyl and 2-thiophenylpyrrolizines by a multicomponent reaction under microwave irradiation using hetarylcarbaldehydes [33]. Similarly, our synthesis (Scheme 2) was carried out by a three-component combinatorial methodology between formylpyrazoles 1, $N$-substituted-maleimides $\mathbf{2}$ and glycine methyl ester $\mathbf{3}$ in refluxing toluene affording the products $\mathbf{4}$ via a double cycloaddition reaction (Table 1).

Scheme 2. Three-component synthesis of pyrazolyldipyrrolo[3,4- $a: 3$ ',4'-f]pyrrolizines 4a-j.

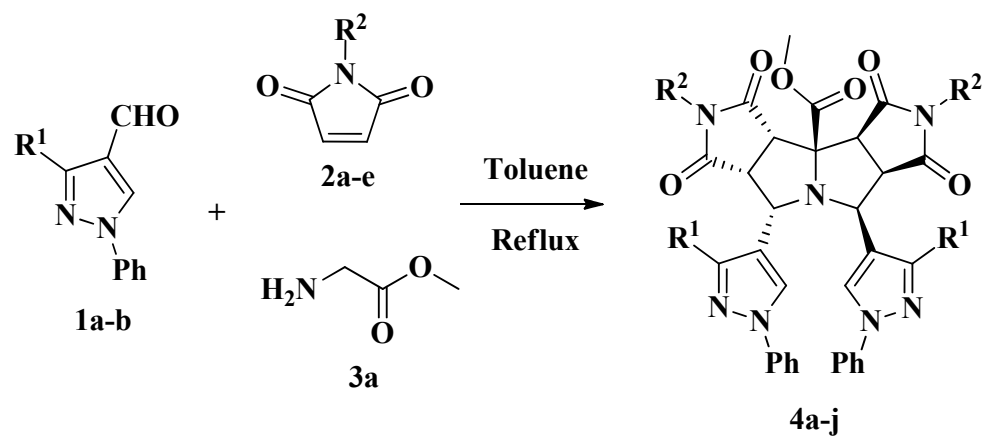

Table 1. Synthesis of diverse pyrazolyldipyrrolo[3,4-a:3',4'-f]pyrrolizines $\mathbf{4 a - j}$.

\begin{tabular}{cccc}
\hline Entry & $\mathbf{R}^{\mathbf{1}}$ & $\mathbf{R}^{\mathbf{2}}$ & Yield (\%) \\
\hline $\mathbf{4 a}$ & $-\mathrm{CH}_{3}$ & $\mathrm{C}_{6} \mathrm{H}_{5}$ & 96 \\
$\mathbf{4 b}$ & $-\mathrm{CH}_{3}$ & $p-\mathrm{ClC}_{6} \mathrm{H}_{4}$ & 73 \\
$\mathbf{4 c}$ & $-\mathrm{CH}_{3}$ & $p-\mathrm{CH}_{3} \mathrm{C}_{6} \mathrm{H}_{4}$ & 84 \\
$\mathbf{4 d}$ & $-\mathrm{CH}_{3}$ & $p-\mathrm{CH}_{3} \mathrm{OC}_{6} \mathrm{H}_{4}$ & 90 \\
$\mathbf{4 e}$ & $-\mathrm{CH}_{3}$ & $\left(3,4-\mathrm{OCH}_{2} \mathrm{O}\right) \mathrm{C}_{6} \mathrm{H}_{3} \mathrm{CH}_{2}$ & 78 \\
$\mathbf{4 f}$ & $\mathrm{C}_{6} \mathrm{H}_{5}$ & $\mathrm{C}_{6} \mathrm{H}_{5}$ & 81 \\
$\mathbf{4 g}$ & $\mathrm{C}_{6} \mathrm{H}_{5}$ & $p-\mathrm{ClC}_{6} \mathrm{H}_{4}$ & 75 \\
$\mathbf{4 h}$ & $\mathrm{C}_{6} \mathrm{H}_{5}$ & $p-\mathrm{CH}_{3} \mathrm{C}_{6} \mathrm{H}_{4}$ & 93 \\
$\mathbf{4 i}$ & $\mathrm{C}_{6} \mathrm{H}_{5}$ & $p-\mathrm{CH}_{3} \mathrm{OC}_{6} \mathrm{H}_{4}$ & 94 \\
$\mathbf{4 j}$ & $\mathrm{C}_{6} \mathrm{H}_{5}$ & $\left(3,4-\mathrm{OCH}_{2} \mathrm{O}\right) \mathrm{C}_{6} \mathrm{H}_{3} \mathrm{CH}_{2}$ & 75 \\
\hline
\end{tabular}

The reaction consists of a domino process as shown in Scheme 3. We propose that initially the condensation of the glycine derivative 3a with the formylpyrazole 1a produced the imine 7a; subsequently, a 1,2-proton shift in imine 7a should afford the azomethine ylide $\mathbf{7 b}$, which in turn should be trapped by the maleimide $\mathbf{2 a}$ to generate the intermediate pyrrolopyrrolidine derivative $\mathbf{8}$ (Scheme 3) [28]. 
Scheme 3. Proposed mechanism for the synthesis of pyrazolyldipyrrolo[3,4- $\left.a: 3^{\prime}, 4^{\prime}-f\right]$ pyrrolizine $4 a$.
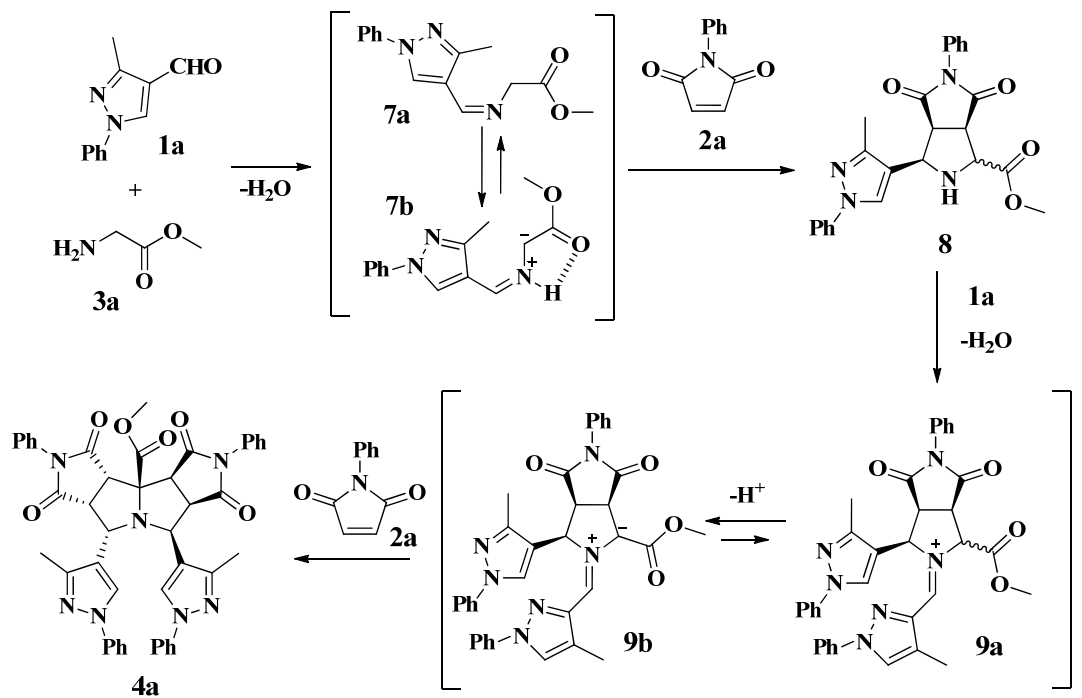

A second condensation should take place between the pyrrolidine intermediate $\mathbf{8}$ and the formylpyrazole 1a affording the iminium ion 9a, which should generate the 1,3-dipolar azomethine ylide $\mathbf{9 b}$ by deprotonation of its acidic proton adjacent to the carbomethoxy group. Finally, the tetracyclic product $\mathbf{4 a}$ would be formed by the diastereoselective 1,3-cycloaddition of a second molecule of maleimide 2a to the ylide $\mathbf{9 b}$. During the process, the formation of the azomethine ylides $\mathbf{7 b}$ and $9 \mathbf{b}$ should be favored by the acidity of the $\alpha$-protons in the moieties 7a and 9a and the in situ stability of these species due to an intramolecular H-bond and $\pi$-conjugation [8,35-37].

The structural elucidation of the new compounds $\mathbf{4 a}-\mathbf{j}$ was made by analysis of the corresponding NMR, infrared and mass spectrometry data. The ${ }^{1} \mathrm{H}-\mathrm{NMR}$ spectra of compounds $\mathbf{4 a}-\mathbf{j}$ show six aliphatic signals corresponding to the protons on the stereogenic centers of the dipyrrolopyrrolizine framework. Two of them appear as triplets corresponding to the H-6a and H-9a protons, and the remaining four appear as doublets assigned to the H-3a, H-3c, H-7 and H-9 protons. Crystals of compound $\mathbf{4 g}$ suitable for single-crystal X-ray diffraction were obtained by slow evaporation from a DMF:EtOH (1:1), thus solution confirming the structure and stereochemistry of the racemic compounds $\mathbf{4 a}-\mathbf{j}$ (Figure 2) [38].

Figure 2. ORTEP drawing of the compound $4 \mathrm{~g}$ with $50 \%$ probability elipsoids. In the ORTEP view of the tetracyclic scaffold, the aryl pendant groups have been removed for the sake of clarity.
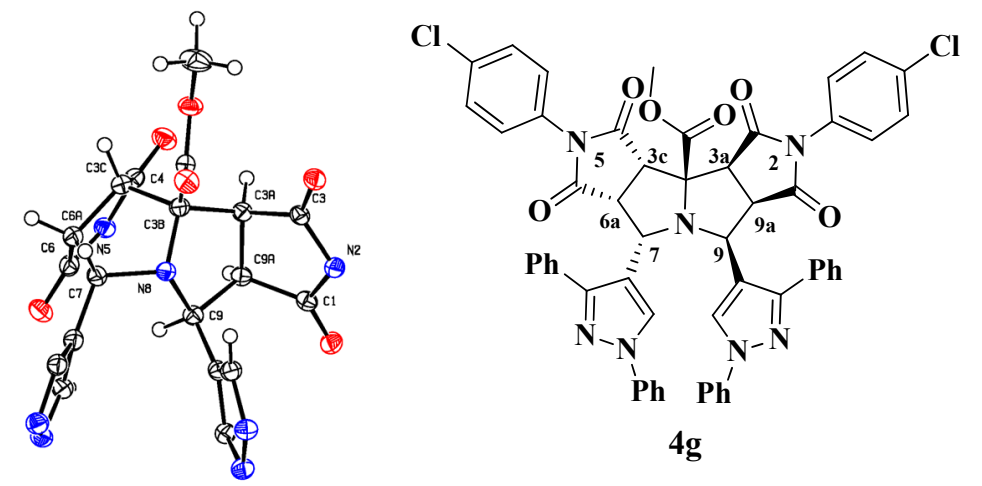


\subsection{Synthesis of Pyrazolylpyrrolo[3,4-c]Pyrroles from N-benzylglicine Ethyl Ester}

On the other hand, it is known that a similar three-component reaction using $\alpha$-amino acids can be stopped at the pyrazolyl-pyrrolo[3,4-c]pyrroles $\mathbf{8}$ if the second cycloaddition is blocked by replacing the proton on the $\alpha$-carbon atom by an alkyl group [28,29]. Thus, in order to preclude the formation of compounds type $\mathbf{4}$, we performed the reaction with $N$-benzylglycine ethyl ester $\mathbf{3} \mathbf{b}$ instead of $\mathbf{3 a}$, along with the formylpyrazoles $1 \mathbf{a}-\mathbf{b}$ and maleimides $\mathbf{2 a - e}$ (Scheme 4). As anticipated, the second amino condensation on the pyrrolidine nitrogen was blocked and the new compounds $5 \mathbf{a}-\mathbf{f}$ and $\mathbf{6 a}-\mathbf{f}$ were obtained in a diastereoselective manner with good yields (Table 2).

Scheme 4. Three-component synthesis of pyrazolylpyrrolo[3,4-c]pyrroles 5 and $\mathbf{6}$.
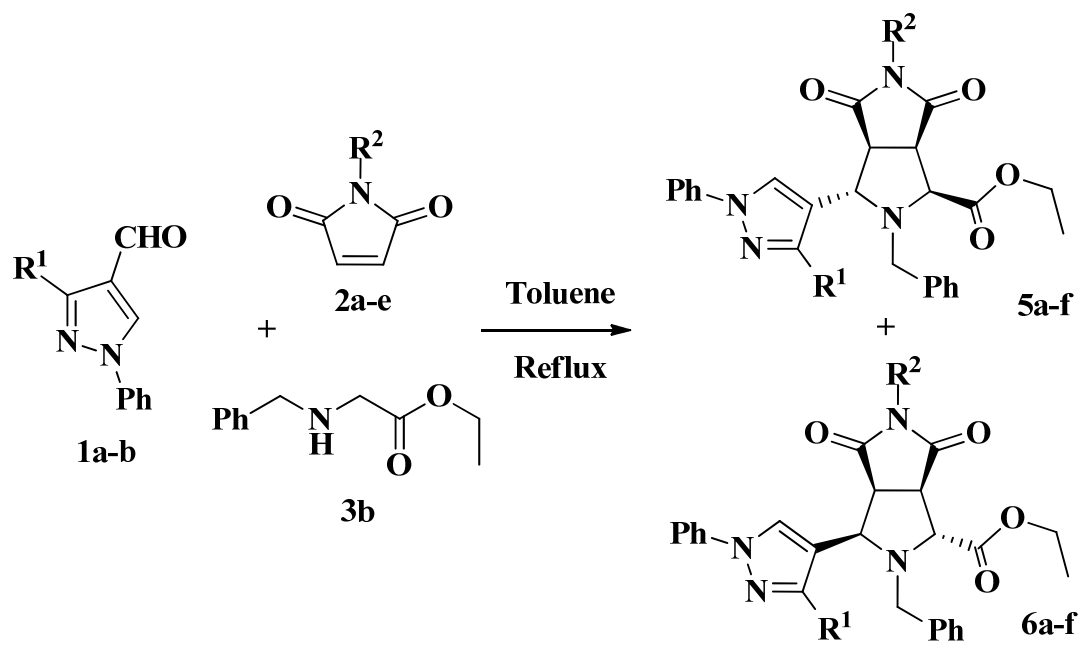

Table 2. Synthesis of diverse pyrazolylpyrrolo[3,4-c]pyrroles 5 and $\mathbf{6}$.

\begin{tabular}{|c|c|c|c|c|}
\hline Entry & $\mathbf{R}^{1}$ & $\mathbf{R}^{2}$ & $d: r^{a}(5: 6)$ & Yield $(\%) 5+6$ \\
\hline 1 & $-\mathrm{CH}_{3}$ & $\mathrm{C}_{6} \mathrm{H}_{5}$ & $1: 7$ & 95 \\
\hline 2 & $-\mathrm{CH}_{3}$ & $p-\mathrm{ClC}_{6} \mathrm{H}_{4}$ & $1: 7$ & 82 \\
\hline 3 & $-\mathrm{CH}_{3}$ & $p-\mathrm{CH}_{3} \mathrm{OC}_{6} \mathrm{H}_{4}$ & $1: 7$ & 96 \\
\hline 4 & $\mathrm{C}_{6} \mathrm{H}_{5}$ & $\mathrm{C}_{6} \mathrm{H}_{5}$ & $1: 7$ & 75 \\
\hline 5 & $\mathrm{C}_{6} \mathrm{H}_{5}$ & $p-\mathrm{ClC}_{6} \mathrm{H}_{4}$ & $1: 7$ & 72 \\
\hline 6 & $\mathrm{C}_{6} \mathrm{H}_{5}$ & $p-\mathrm{CH}_{3} \mathrm{OC}_{6} \mathrm{H}_{4}$ & $1: 7$ & 90 \\
\hline
\end{tabular}

In this approach, the first step is a condensation between the formylpyrazole 1a and the $N$-benzyl glycine ethyl ester $\mathbf{3 b}$ affording the iminium ion $\mathbf{1 0 a}$, which is subsequently deprotonated giving azomethine ylide $\mathbf{1 0 b}$ (Scheme 5). Then, the 1,3-dipolar cycloaddition of $\mathbf{1 0 b}$ with the $N$-phenyl maleimide 2a afforded the diastereomers 5a and 6a. In all cases, the reaction showed good diastereoselectivity toward isomers $\mathbf{6}$, in which repulsive interactions between the ester group on the C-1 and C-6 carbonyl group are avoided due to the trans configuration between them as shown in 6 a (Scheme 5) [17]. 
Scheme 5. Proposed mechanism for the synthesis of pyrazolylpyrrolo[3,4-c]pyrroles 5a and 6a.

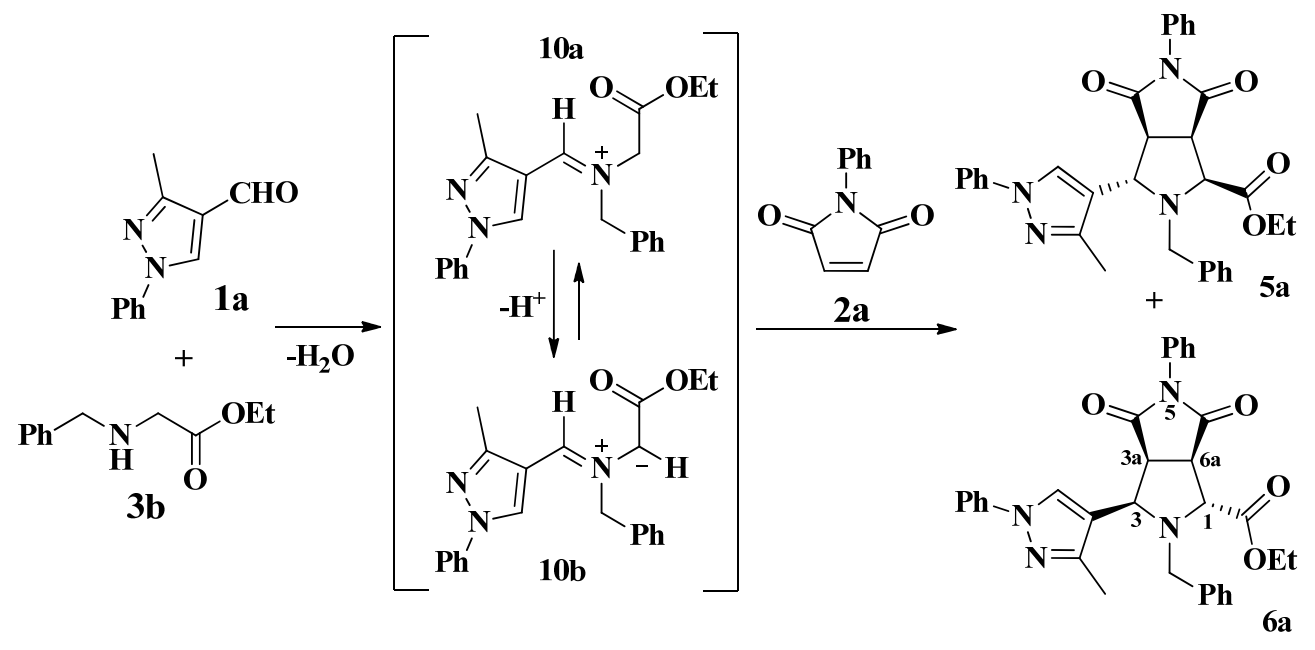

According to the ${ }^{1} \mathrm{H}-\mathrm{NMR}$ analysis of compounds 6 the H-1 proton on the stereogenic center appears as a singlet due to the dihedral angle (aprox. 90 $)$ with the H-6a proton indicating a trans configuration. On the other hand, both the H-3 and H-6a protons appear as doublets with coupling constants about $9.0 \mathrm{~Hz}$ corresponding to a cis configuration with the $\mathrm{H}-3$ a proton, which in turn appears as a double doublet. Meantime, in compounds 5 the $\mathrm{H}-1$ proton is observed as a doublet with a coupling constant $J \approx 9.0 \mathrm{~Hz}$ because of its cis configuration with respect to the $\mathrm{H}-6$ a proton, while the $\mathrm{H}-3$ proton appears as a doublet with a smaller coupling constant $(J \approx 5.5 \mathrm{~Hz})$ due to a trans configuration with respect to the H-3a proton [39].

\subsection{Theoretical Calculations}

To confirm our findings about the stereochemistry of the reactions as well as the obtained compounds 4-6, theoretical calculations were carried out with the DFT approach using the B.01 revision of the Gaussian 09 program package [40]. DFT calculations were performed using Becke's three-parameter B3LYP exchange-correlation functional and the $6-311 \mathrm{G}++$ basis set. The geometry of compound 6d was theoretically optimized and the most stable configuration is depicted in Figure 3. Although the found energy values for the cis and trans configurations of the stereoisomers $\mathbf{5 d}$ and $\mathbf{6 d}$ are very close, the trans configuration for $\mathbf{6 d}$ is slightly favored by $7.82696 \times 10^{-17} \mathrm{Kcal}$ over the cis form for 5d. This finding is in agreement with the Karplus theory [41] and with our experimental ${ }^{1} \mathrm{H}-\mathrm{NMR}$ measurements, since the dihedral angle $1 \mathrm{H}-\mathrm{C}-\mathrm{C}-6 \mathrm{aH}$ for the stereoisomer $6 \mathbf{d}$ is $-94.41^{\circ}$ and therefore in its ${ }^{1} \mathrm{H}-\mathrm{NMR}$ spectrum the coupling constant between the H-1 and H-6a protons has a value $J_{3} \sim 0.0 \mathrm{~Hz}$.

In order to verify the accuracy of the theoretical method, the geometry of compound $\mathbf{4 g}$ was theoretically optimized and the most stable configuration is depicted in Figure 4. Geometrical parameters such as bond length and molecular angles were calculated. We observed that the calculated parameters were very close to the experimental values, measured by X-ray diffraction. Some of these geometrical stocks are listed in Table 3. 
Figure 3. Minimum-energy configuration calculated for the compound $\mathbf{6 d}$ at the B3LYP/ 6-311G++ level. Dihedral Angle $(1 \mathrm{H}-\mathrm{C}-\mathrm{C}-6 \mathrm{aH})=-94.41^{\circ}$.

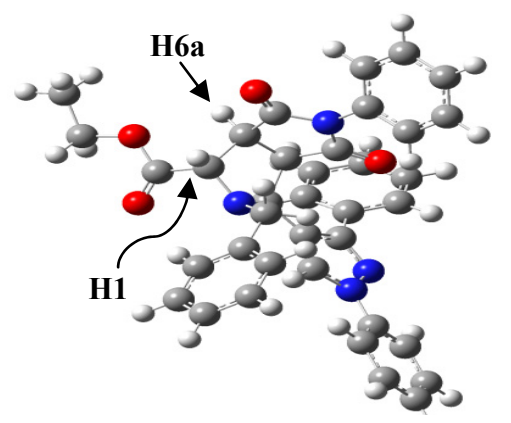

Figure 4. Minimum-energy configuration calculated for compound $\mathbf{4 g}$ at the B3LYP/ 6-311G++ level.

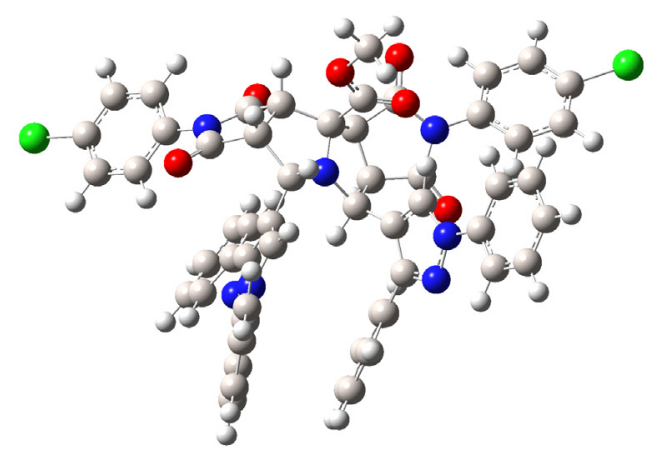

Table 3. Geometrical parameters of compound 4g; X-ray data is compared with theoretical calculated parameters.

\begin{tabular}{cccccc}
\hline Bond & $\begin{array}{c}\text { X-Ray } \\
\text { Length }(\boldsymbol{\AA} \AA)\end{array}$ & $\begin{array}{c}\text { Calculated } \\
\text { Length }(\boldsymbol{\AA})\end{array}$ & $\begin{array}{c}\text { Angle } \\
\text { Atoms }\end{array}$ & X-Ray Angle $\left(^{\circ}\right)$ & Calculated Angle $\left(^{\circ}\right)$ \\
\hline C1-O1 & $1.210(2)$ & 1.213 & O1-C1-N2 & $124.61(17)$ & 125.02 \\
C1-N2 & $1.393(2)$ & 1.398 & O1-C1-C8a & $128.00(16)$ & 127.64 \\
C1-C8a & $1.508(3)$ & 1.512 & N2-C1-C8a & $107.36(14)$ & 110.73 \\
N2-C3 & $1.399(2)$ & 1.409 & C1-N2-C3 & $112.48(15)$ & 114.34 \\
C3-O3 & $1.209(2)$ & 1.211 & C1-N2-C21 & $122.84(14)$ & 120.21 \\
C3-C3a & $1.529(3)$ & 1.534 & C3-N2-C21 & $124.47(15)$ & 124.88 \\
C3c-C4 & $1.507(3)$ & 1.511 & O3-C3-N2 & $124.53(17)$ & 123.00 \\
\hline
\end{tabular}

\section{Experimental}

\subsection{General Information}

The pyrazole-4-carbaldehydes $\mathbf{1 a}-\mathbf{b}$ and the glycine ester derivates $\mathbf{3 a}-\mathbf{b}$ (analytical reagent grade) were purchased from Aldrich (St. Louis, Missouri, United State), Fluka (St. Louis, MO, USA) and Merck (Darmstadt, Alemania) and were used without further purification. The maleimides $\mathbf{2 a - e}$ were obtained according to the already reported procedure [42]. Solvents and other chemicals commercially available were used as shipped. Silica gel aluminium plates (Merck $60 \mathrm{~F}_{254}$ ) were used for analytical TLC. Melting points were taken on Stuart SMP10 Melting point apparatus and are uncorrected. IR 
spectra were recorded on a Shimadzu FTIR 8400 spectrophotometer using $\mathrm{KBr}$ disks or $\mathrm{CH}_{2} \mathrm{Cl}_{2}$ as solvent. ${ }^{1} \mathrm{H}$ - and ${ }^{13} \mathrm{C}$-NMR were recorded on a Bruker Avance 400 spectrometer operating at 400 and $100 \mathrm{MHz}$ respectively, using $\mathrm{CDCl}_{3}$ as solvent. Mass spectra were obtained on a Shimadzu GCMS-QP 2010 spectrometer (equipped with a direct inlet probe) operating at $70 \mathrm{eV}$ and with a Bruker Esquire 6000 spectrometer equipped with an electrospray ionization source and an ion-trap detector. Microanalyses were performed on a LECO CHNS-900 elemental analyzer and the values are within $\pm 0.4 \%$ of theoretical values.

\subsection{Synthesis and Characterization Data for Pyrazolyldipyrrolo[3,4-a:3',4'-f]Pyrrolizines $\mathbf{4 a - j}$}

General Synthetic Procedure

To a $25.0 \mathrm{~mL}$ round bottom flask equipped with a magnetic stirring bar and a reflux condenser were added pyrazole-4-carboxaldehyde $\mathbf{1 a}-\mathbf{b}(0.2 \mathrm{mmol}), N$-substituted-maleimide $\mathbf{2 a}-\mathbf{e}(0.2 \mathrm{mmol})$, glycine methyl ester $3 \mathbf{a}(0.2 \mathrm{mmol})$ and toluene $(8 \mathrm{~mL})$. The mixture was heated under reflux for $8-10 \mathrm{~h}$. The reaction mixture was cooled to ambient temperature and the resulting precipitate was collected by filtration and washed with hexane-toluene (1:1) to obtain the pure compounds. In some cases, the solid was recrystallized from a mixture ethanol-DMF (1:1) to obtain the pure compound 4.

Methyl 7,9-bis(3-methyl-1-phenyl-1H-pyrazol-4-yl)-1,3,4,6-tetraoxo-2,5-diphenyldodecahydro-1Hdipyrrolo[3,4-a:3',4'-f]pyrrolizine-3b-carboxylate (4a). Beige solid. Yield: 96\%; m.p.: 182-184 ${ }^{\circ} \mathrm{C}$. IR (KBr): v 3473, 2954, 1753, $1715 \mathrm{~cm}^{-1} .{ }^{1} \mathrm{H}-\mathrm{NMR}\left(400 \mathrm{MHz}, \mathrm{CDCl}_{3}\right) \delta$ ppm: 2.01 (s, 3H), 2.26 (s, 3H), $3.51(\mathrm{dd}, J=8.0 \mathrm{~Hz} ; J=8.3 \mathrm{~Hz}, 1 \mathrm{H}), 3.74(\mathrm{t}, J=8.0 \mathrm{~Hz}, 1 \mathrm{H}), 3.99$ (s, 3H), 4.29 (d, $J=8.3 \mathrm{~Hz}$, $1 \mathrm{H}), 4.46(\mathrm{~d}, J=10.5 \mathrm{~Hz}, 1 \mathrm{H}), 4.64(\mathrm{~d}, J=8.3 \mathrm{~Hz}, 1 \mathrm{H}), 4.91(\mathrm{~d}, J=7.8 \mathrm{~Hz}, 1 \mathrm{H}), 6.81(\mathrm{~d}, J=8.8 \mathrm{~Hz}$, 2H), $7.18(\mathrm{~d}, J=8.5 \mathrm{~Hz}, 2 \mathrm{H}), 7.20-7.26(\mathrm{~m}, 2 \mathrm{H}), 7.28-7.39(\mathrm{~m}, 12 \mathrm{H}), 7.53(\mathrm{~s}, 1 \mathrm{H}), 7.57$ $(\mathrm{d}, J=8.8 \mathrm{~Hz}, 2 \mathrm{H}), 7.98(\mathrm{~s}, 1 \mathrm{H}) .{ }^{13} \mathrm{C}-\mathrm{NMR}\left(100 \mathrm{MHz}, \mathrm{CDCl}_{3}\right) \delta \mathrm{ppm}: 11.5\left(\mathrm{CH}_{3}\right), 11.9\left(\mathrm{CH}_{3}\right), 48.2$ $(\mathrm{CH}), 48.9(\mathrm{CH}), 49.5(\mathrm{CH}), 53.2(\mathrm{CH}), 53.5\left(\mathrm{OCH}_{3}\right), 58.1(\mathrm{CH}), 60.6(\mathrm{CH}), 79.34(\mathrm{C}), 112.2(\mathrm{C})$, $118.4(\mathrm{CH}), 118.8(\mathrm{CH}), 119.4(\mathrm{CH}), 120.1(\mathrm{C}), 125.9(\mathrm{CH}), 126.3(\mathrm{CH}), 126.4(\mathrm{CH}), 126.8(\mathrm{CH})$, $126.9(\mathrm{CH}), 127.3(\mathrm{CH}), 129.1(\mathrm{CH}), 129.2(\mathrm{CH}), 129.3(\mathrm{CH}), 129.40(\mathrm{CH}), 130.0(\mathrm{CH}), 134.3(\mathrm{C})$, 135.4 (C), 139.6 (C), 139.7 (C), 146.6 (C), 150.7 (C), 170.2 (C), 171.7 (C), 173.2 (C), 174.7 (C), 175.7 (C). MS (EI, $70 \mathrm{eV)} \mathrm{m/z} \mathrm{( \% ):} 771\left(\mathrm{M}^{+}, 2\right), 712$ (13), 598 (100), 539 (70), 268 (27), 211 (32), 171 (75). Elemental Analyses calcd. for $\mathrm{C}_{45} \mathrm{H}_{37} \mathrm{~N}_{7} \mathrm{O}_{6} .2 \mathrm{H}_{2} \mathrm{O}: \mathrm{C}: 66.90, \mathrm{H}: 5.12, \mathrm{~N}: 12.14$. Found: C: 66.75, H: 5.47, N: 12.07 .

Methyl 2,5-bis(4-chlorophenyl)-7,9-bis(3-methyl-1-phenyl-1H-pyrazol-4-yl)-1,3,4,6 tetraoxododecahydro-1H-dipyrrolo[3,4-a:3',4'-f]pyrrolizine-3b-carboxylate (4b). Pale yellow solid. Yield: 73\%; m.p.: 170-172 ${ }^{\circ} \mathrm{C}$. IR (KBr): v 3479, 2953, $1716 \mathrm{~cm}^{-1} .{ }^{1} \mathrm{H}-\mathrm{NMR}\left(400 \mathrm{MHz}, \mathrm{CDCl}_{3}\right) \delta \mathrm{ppm}: 2.03$ (s, 3H), $2.29(\mathrm{~s}, 3 \mathrm{H}), 3.53(\mathrm{dd}, J=10.7,8.2,1 \mathrm{H}), 3.76(\mathrm{t}, J=8.0 \mathrm{~Hz}, 1 \mathrm{H}), 4.02(\mathrm{~s}, 3 \mathrm{H}), 4.31(\mathrm{~d}, J=8.3 \mathrm{~Hz}, 1 \mathrm{H})$, $4.48(\mathrm{~d}, J=10.5 \mathrm{~Hz}, 1 \mathrm{H}), 4.66(\mathrm{~d}, J=8.3 \mathrm{~Hz}, 1 \mathrm{H}), 4.94(\mathrm{~d}, J=7.8 \mathrm{~Hz}, 1 \mathrm{H}), 6.83(\mathrm{~d}, J=8.8 \mathrm{~Hz}, 2 \mathrm{H})$, $7.21(\mathrm{~d}, J=8.5 \mathrm{~Hz}, 2 \mathrm{H}), 7.26(\mathrm{~m}, 2 \mathrm{H}), 7.37(\mathrm{~m}, 10 \mathrm{H}), 7.55(\mathrm{~s}, 1 \mathrm{H}), 7.60(\mathrm{~d}, J=8.8 \mathrm{~Hz}, 2 \mathrm{H}), 8.00(\mathrm{~s}$, 1H). ${ }^{13} \mathrm{C}-\mathrm{NMR}\left(100 \mathrm{MHz}, \mathrm{CDCl}_{3}\right) \delta \mathrm{ppm}: 11.5\left(\mathrm{CH}_{3}\right), 11.9\left(\mathrm{CH}_{3}\right), 48.2(\mathrm{CH}), 48.9(\mathrm{CH}), 49.5(\mathrm{CH})$, $53.2(\mathrm{CH}) ; 53.5\left(\mathrm{OCH}_{3}\right), 58.1(\mathrm{CH}), 60.6(\mathrm{CH}), 79.3(\mathrm{C}), 112.2(\mathrm{C}) 118.8(\mathrm{CH}), 119.4(\mathrm{CH}), 120.1(\mathrm{C})$, $125.9(\mathrm{CH}), 126.3(\mathrm{CH}), 126.4(\mathrm{CH}), 126.8(\mathrm{CH}), 126.9(\mathrm{CH}), 127.3(\mathrm{CH}), 129.1(\mathrm{CH}), 129.2(\mathrm{CH})$, 
$129.3(\mathrm{CH}), 129.4(\mathrm{C}), 129.7(\mathrm{C}), 130.0(\mathrm{CH}), 134.3(\mathrm{C}), 135.4(\mathrm{C}), 139.6(\mathrm{C}), 139.7$ (C), $146.6(\mathrm{C})$, 150.7 (C), 170.2 (C), 171.7 (C), 173.2 (C), 174.7 (C), 175.7 (C). MS (EI, $70 \mathrm{eV)} \mathrm{m/z} \mathrm{( \% ):} 839$ (M , 2), 780 (18), 632 (42), 268 (22), 211 (39), 171 (100). Elemental Analyses calcd. for $\mathrm{C}_{45} \mathrm{H}_{35} \mathrm{Cl}_{2} \mathrm{~N}_{7} \mathrm{O}_{6} \cdot \mathrm{H}_{2} \mathrm{O}$ : C: 62.94, H: 4.34, N: 11.42. Found: C: 63.32, H: 4.36, N: 11.34.

Methyl 7,9-bis(3-methyl-1-phenyl-1H-pyrazol-4-yl)-1,3,4,6-tetraoxo-2,5-di-p-tolyldodecahydro-1Hdipyrrolo[3,4-a:3',4'-f]pyrrolizine-3b-carboxylate (4c). Beige solid. Yield: 84\%; m.p.: 179-181 ${ }^{\circ} \mathrm{C}$. IR (KBr): v 3647, 2952, 1784, 1756, $1696 \mathrm{~cm}^{-1} .{ }^{1} \mathrm{H}-\mathrm{NMR}\left(400 \mathrm{MHz}, \mathrm{CDCl}_{3}\right) \delta$ ppm: 2.02 (s, 3H), 2.25 (s, 3H), 2.27 (s, 3H), $2.46(\mathrm{~s}, 3 \mathrm{H}), 3.56(\mathrm{dd}, J=10.7,8.2 \mathrm{~Hz}, 1 \mathrm{H}), 3.71-3.77(\mathrm{~m}, 1 \mathrm{H}), 4.00(\mathrm{~s}, 3 \mathrm{H})$, $4.28(\mathrm{~d}, J=8.3 \mathrm{~Hz}, 1 \mathrm{H}), 4.51(\mathrm{~d}, J=10.8 \mathrm{~Hz}, 1 \mathrm{H}), 4.66(\mathrm{~d}, J=8.3 \mathrm{~Hz}, 1 \mathrm{H}), 4.93(\mathrm{~d}, J=8.0 \mathrm{~Hz}, 1 \mathrm{H})$, $6.75(\mathrm{~d}, J=8.3 \mathrm{~Hz}, 2 \mathrm{H}), 7.03(\mathrm{~d}, J=7.5 \mathrm{~Hz}, 2 \mathrm{H}), 7.21-7.25(\mathrm{~m}, 4 \mathrm{H}), 7.33-7.39(\mathrm{~m}, 5 \mathrm{H}), 7.39-7.44(\mathrm{~m}$, $5 \mathrm{H}), 7.58(\mathrm{~s}, 1 \mathrm{H}), 8.04(\mathrm{~s}, 1 \mathrm{H}) .{ }^{13} \mathrm{C}-\mathrm{NMR}\left(100 \mathrm{MHz}, \mathrm{CDCl}_{3}\right) \delta \mathrm{ppm}: 11.5\left(\mathrm{CH}_{3}\right), 11.9\left(\mathrm{CH}_{3}\right), 21.0$ $\left(\mathrm{CH}_{3}\right), 21.3\left(\mathrm{CH}_{3}\right), 48.2(\mathrm{CH}), 48.8(\mathrm{CH}), 49.6(\mathrm{CH}), 53.3(\mathrm{CH}), 53.4\left(\mathrm{OCH}_{3}\right), 58.1(\mathrm{CH})$, $60.5(\mathrm{CH}), 79.3(\mathrm{C}), 112.5(\mathrm{C}), 118.4(\mathrm{CH}), 118.9(\mathrm{CH}), 119.4(\mathrm{CH}), 120.3(\mathrm{C}), 125.4(\mathrm{CH})$, $125.5(\mathrm{CH}), 125.7(\mathrm{CH}), 126.1(\mathrm{CH}), 126.3(\mathrm{CH}), 127.5(\mathrm{CH}), 128.4(\mathrm{C}), 128.6(\mathrm{C}), 129.2(\mathrm{CH})$, $129.6(\mathrm{CH}), 130.4(\mathrm{CH}), 138.7(\mathrm{C}), 139.6(\mathrm{C}), 139.7(\mathrm{C}), 139.8(\mathrm{C}), 146.7(\mathrm{C}), 150.7$ (C), $170.4(\mathrm{C})$, 172.2 (C), 173.7 (C), 175.1 (C), 176.2 (C). MS (EI, 70 eV) m/z (\%): 799 (M+, 2), 740 (8), 612 (100), 553 (18), 257 (60), 197 (75). Elemental Analyses calcd. for $\mathrm{C}_{47} \mathrm{H}_{41} \mathrm{~N}_{7} \mathrm{O}_{6}$ : C: 70.57, H: 5.17, N: 12.26. Found: C: $70.87, \mathrm{H}: 5.41, \mathrm{~N}: 11.90$.

Methyl 2,5-bis(4-methoxyphenyl)-7,9-bis(3-methyl-1-phenyl-1H-pyrazol-4-yl)-1,3,4,6-tetraoxododecahydro-1H-dipyrrolo[3,4-a:3',4'-f]pyrrolizine-3b-carboxylate (4d). White Solid. Yield: 90\%; m.p.: 174-176 ${ }^{\circ} \mathrm{C}$. IR (KBr): v 3478, 2955, 1714, $1598 \mathrm{~cm}^{-1} .{ }^{1} \mathrm{H}-\mathrm{NMR}\left(400 \mathrm{MHz}, \mathrm{CDCl}_{3}\right) \delta \mathrm{ppm}: 2.02(\mathrm{~s}$, $3 \mathrm{H}), 2.27(\mathrm{~s}, 3 \mathrm{H}), 3.54(\mathrm{dd}, J=10.7,8.2 \mathrm{~Hz}, 1 \mathrm{H}), 3.68-3.75(\mathrm{~m}, 4 \mathrm{H}), 3.88(\mathrm{~s}, 3 \mathrm{H}), 4.00(\mathrm{~s}, 3 \mathrm{H}), 4.26$ $(\mathrm{d}, J=8.3 \mathrm{~Hz}, 1 \mathrm{H}), 4.50(\mathrm{~d}, J=10.5 \mathrm{~Hz}, 1 \mathrm{H}), 4.65(\mathrm{~d}, J=8.0 \mathrm{~Hz}, 1 \mathrm{H}), 4.92(\mathrm{~d}, J=7.8 \mathrm{~Hz}, 1 \mathrm{H}), 6.72$ $(\mathrm{d}, J=9.0 \mathrm{~Hz}, 2 \mathrm{H}), 6.75(\mathrm{~d}, J=9.0,2 \mathrm{H}), 7.10(\mathrm{~d}, J=9.0 \mathrm{~Hz}, 2 \mathrm{H}), 7.20-7.29(\mathrm{~m}, 4 \mathrm{H}), 7.30-7.44(\mathrm{~m}$, $8 \mathrm{H}), 7.57(\mathrm{~s}, 1 \mathrm{H}), 8.05(\mathrm{~s}, 1 \mathrm{H}) .{ }^{13} \mathrm{C}-\mathrm{NMR}\left(100 \mathrm{MHz}, \mathrm{CDCl}_{3}\right) \delta \mathrm{ppm}: 11.5\left(\mathrm{CH}_{3}\right), 11.9\left(\mathrm{CH}_{3}\right), 48.2$ $(\mathrm{CH}), 48.8(\mathrm{CH}), 49.6(\mathrm{CH}), 53.3(\mathrm{CH}), 53.4\left(\mathrm{OCH}_{3}\right), 55.3\left(\mathrm{OCH}_{3}\right), 55.6\left(\mathrm{OCH}_{3}\right), 58.1(\mathrm{CH}), 60.5(\mathrm{CH})$, $79.2(\mathrm{C}), 112.5(\mathrm{C}), 114.3(\mathrm{CH}), 115.1(\mathrm{CH}), 118.9(\mathrm{CH}), 119.4(\mathrm{CH}), 120.4(\mathrm{C}), 123.7(\mathrm{C}), 123.8(\mathrm{C})$, $126.1(\mathrm{CH}), 126.3(\mathrm{CH}), 126.8(\mathrm{CH}), 126.9(\mathrm{CH}), 127.5(\mathrm{CH}), 129.2(\mathrm{CH}), 139.7(\mathrm{C}), 139.8(\mathrm{C}), 146.7(\mathrm{C})$, 150.7 (C), 159.3 (C), 160.1 (C), 170.4 (C), 172.3 (C), 173.8 (C), 175.2 (C), 176.3 (C). MS (EI, 70 eV) m/z (\%): $831\left(\mathrm{M}^{+}, 2\right), 772$ (8), 628 (67), 268 (43), 203 (46), 171 (100). Elemental Analyses calcd. for $\mathrm{C}_{47} \mathrm{H}_{45} \mathrm{~N}_{7} \mathrm{O}_{10}: \mathrm{C}: 65.04, \mathrm{H}: 5.23, \mathrm{~N}: 11.30$. Found: C: 65.44, H: 4.90, N: 11.27.

Methyl 2,5-bis(benzo[d][1,3]dioxol-5-ylmethyl)-7,9-bis(3-methyl-1-phenyl-1H-pyrazol-4-yl)-1,3,4,6tetraoxododecahydro-1H-dipyrrolo[3,4-a:3',4'-f]pyrrolizine-3b-carboxylate (4e). Beige Solid. Yield: 78\%; m.p.: 243-245 ${ }^{\circ} \mathrm{C}$. IR (KBr): v 3459, 2898, 1749, 1694, $1598 \mathrm{~cm}^{-1} .{ }^{1} \mathrm{H}-\mathrm{NMR}\left(400 \mathrm{MHz}, \mathrm{CDCl}_{3}\right)$ $\delta$ ppm: $1.56(\mathrm{~s}, 3 \mathrm{H}), 2.11(\mathrm{~s}, 3 \mathrm{H}), 2.91(\mathrm{dd}, J=10.5,8.5 \mathrm{~Hz}, 1 \mathrm{H}), 3.51(\mathrm{t}, J=8.4 \mathrm{~Hz}, 1 \mathrm{H}), 3.65(\mathrm{~d}$, $J=10.5 \mathrm{~Hz}, 1 \mathrm{H}), 3.95(\mathrm{~s}, 3 \mathrm{H}), 4.22(\mathrm{~d}, J=8.3 \mathrm{~Hz}, 1 \mathrm{H}), 4.23-4.34(\mathrm{~m}, 2 \mathrm{H}), 4.49(\mathrm{~d}, J=8.5 \mathrm{~Hz}, 1 \mathrm{H})$, 4.63-4.74 (m, 3H), 5.71-5.83 (m, 2H), 5.86-5.96 (m, 2H), $6.47(\mathrm{~d}, J=7.0 \mathrm{~Hz}, 1 \mathrm{H}), 6.63(\mathrm{~d}, J=7.3 \mathrm{~Hz}$, $1 \mathrm{H}), 6.70(\mathrm{~s}, 1 \mathrm{H}), 6.80(\mathrm{~d}, J=7.8 \mathrm{~Hz}, 1 \mathrm{H}), 6.87-7.01(\mathrm{~m}, 1 \mathrm{H}), 7.08-7.11(\mathrm{~m}, 1 \mathrm{H}), 7.12(\mathrm{~d}, J=1.5 \mathrm{~Hz}$, 1H), 7.15-7.23 (m, 5H), 7.27-7.37 (m, 5H), $7.52(\mathrm{~s}, 1 \mathrm{H}) .{ }^{13} \mathrm{C}-\mathrm{NMR}\left(100 \mathrm{MHz}, \mathrm{CDCl}_{3}\right) \delta \mathrm{ppm}$ : $11.2\left(\mathrm{CH}_{3}\right)$, 
$11.3\left(\mathrm{CH}_{3}\right), 42.2\left(\mathrm{CH}_{2}\right), 42.9\left(\mathrm{CH}_{2}\right), 48.6(\mathrm{CH}), 48.7(\mathrm{CH}), 48.8(\mathrm{CH}), 52.5(\mathrm{CH}), 53.4\left(\mathrm{OCH}_{3}\right), 59.7$ $(\mathrm{CH}), 63.6(\mathrm{CH}), 79.3(\mathrm{C}), 101.0\left(\mathrm{CH}_{2}\right), 101.5\left(\mathrm{CH}_{2}\right), 108.1(\mathrm{CH}), 108.9(\mathrm{CH}), 109.1(\mathrm{CH}), 109.6$ $(\mathrm{CH}), 118.5(\mathrm{CH}), 119.2(\mathrm{CH}), 122.3(\mathrm{CH}), 122.4(\mathrm{CH}), 123.3(\mathrm{CH}), 125.5(\mathrm{CH}), 125.6(\mathrm{CH}), 126.2$ $(\mathrm{CH}), 128.1(\mathrm{C}), 128.8(\mathrm{C}), 129.0(\mathrm{CH}), 129.2(\mathrm{CH}), 139.6(\mathrm{C}), 139.7(\mathrm{C}), 147.1(\mathrm{C}), 147.5(\mathrm{C}), 148.2$ (C), 148.4 (C), 150.2 (C), 170.7 (C), 172.8 (C), 174.7 (C), 175.8 (C), 176.4 (C). MS (EI, 70 eV) $\mathrm{m} / \mathrm{z}$ (\%): $887\left(\mathrm{M}^{+}, 1\right), 657$ (20), 656 (50), 231 (16), 171 (74), 135 (100). Elemental Analyses calcd. for $\mathrm{C}_{49} \mathrm{H}_{41} \mathrm{~N}_{7} \mathrm{O}_{10} .2 \mathrm{H}_{2} \mathrm{O}: \mathrm{C}: 63.70, \mathrm{H}: 4.91, \mathrm{~N}: 10.61$. Found: C: 64.03, H: 5.15, N: 10.82.

Methyl 7,9-bis(1,3-diphenyl-1H-pyrazol-4-yl)-1,3,4,6-tetraoxo-2,5-diphenyldodecahydro-1H-dipyrrolo[3,4-a:3',4'-f]pyrrolizine-3b-carboxylate (4f). Yellow Solid. Yield: 81\%; m.p.: 284-286 ${ }^{\circ} \mathrm{C}$. IR (KBr): v 3062, 2954, 1746, 1712, $1598 \mathrm{~cm}^{-1}$. ${ }^{1} \mathrm{H}-\mathrm{NMR}\left(400 \mathrm{MHz}, \mathrm{CDCl}_{3}-d\right) \delta \mathrm{ppm}: 3.59$ (t, $\left.J=8.4 \mathrm{~Hz}, 1 \mathrm{H}\right)$, $3.78-3.85(\mathrm{~m}, 1 \mathrm{H}), 3.97(\mathrm{~s}, 3 \mathrm{H}), 4.36(\mathrm{~d}, J=8.5 \mathrm{~Hz}, 1 \mathrm{H}), 4.72(\mathrm{~d}, J=10.5 \mathrm{~Hz}, 1 \mathrm{H}), 4.82(\mathrm{~d}, J=8.3 \mathrm{~Hz}$, $1 \mathrm{H}), 5.15(\mathrm{~d}, J=8.5 \mathrm{~Hz}, 1 \mathrm{H}), 6.97(\mathrm{dd}, J=6.5,2.8 \mathrm{~Hz}, 2 \mathrm{H}), 7.02-7.08(\mathrm{~m}, 2 \mathrm{H}), 7.11-7.17(\mathrm{~m}, 3 \mathrm{H})$, 7.21-7.25 (m, 5H), 7.28-7.35 (m, 7H), 7.37-7.51 (m, 7H), 7.53-7.59 (m, 3H), $7.83(\mathrm{~s}, 1 \mathrm{H}), 7.91(\mathrm{~d}$, $J=7.3 \mathrm{~Hz}, 2 \mathrm{H}) .{ }^{13} \mathrm{C}-\mathrm{NMR}\left(100 \mathrm{MHz}, \mathrm{CDCl}_{3}\right) \delta \mathrm{ppm}$ : $48.7(\mathrm{CH}), 49.5(\mathrm{CH}), 49.6(\mathrm{CH}), 52.9(\mathrm{CH}), 53.7$ $\left(\mathrm{OCH}_{3}\right), 59.2(\mathrm{CH}), 60.7(\mathrm{CH}), 80.1(\mathrm{C}), 111.0(\mathrm{C}), 119.3(\mathrm{CH}), 119.8(\mathrm{CH}), 120.3(\mathrm{C}), 125.7(\mathrm{CH})$, $126.1(\mathrm{CH}), 126.5(\mathrm{CH}), 126.7(\mathrm{CH}), 127.1(\mathrm{CH}), 127.9(\mathrm{CH}), 128.0(\mathrm{CH}), 128.2(\mathrm{CH}), 128.3(\mathrm{CH})$, $128.5(\mathrm{CH}), 128.6(\mathrm{CH}), 128.6(\mathrm{CH}), 129.0(\mathrm{CH}), 129.1(\mathrm{CH}), 129.2(\mathrm{CH}), 129.3(\mathrm{CH}), 129.4(\mathrm{CH})$, $129.8(\mathrm{CH}), 131.1(\mathrm{C}), 131.2(\mathrm{C}), 132.3(\mathrm{C}), 132.5$ (C), 139.6 (C), $139.6(\mathrm{C}), 150.5$ (C), $152.7(\mathrm{C})$, 170.1 (C), 172.5 (C), 173.7 (C), 175.3 (C), 176.0 (C). MS (EI, $70 \mathrm{eV}) \mathrm{m} / z(\%): 895\left(\mathrm{M}^{+}, 1\right), 836(6), 722$ (71), 298 (23), 273 (27), 233 (100), 173 (42). Elemental Analyses calcd. for $\mathrm{C}_{55} \mathrm{H}_{45} \mathrm{~N}_{7} \mathrm{O}_{6} \cdot 3 \mathrm{H}_{2} \mathrm{O}: \mathrm{C}$ : 69.54, H: 4.99, N: 10.32. Found: C: 69.43, H: 4.94, N: 10.42.

Methyl 2,5-bis(4-chlorophenyl)-7,9-bis(1,3-diphenyl-1H-pyrazol-4-yl)-1,3,4,6-tetraoxododecahydro1H-dipyrrolo[3,4-a:3',4'-f]pyrrolizine-3b-carboxylate (4g). White Solid. Yield: 75\%; m.p.: 275-277 ${ }^{\circ} \mathrm{C}$. IR (KBr): v 3447, 1718, 1598, $1496 \mathrm{~cm}^{-1} .{ }^{1} \mathrm{H}-\mathrm{NMR}\left(400 \mathrm{MHz}, \mathrm{CDCl}_{3}\right) \delta$ ppm: 3.50-365 (m, 1H); $3.72-3.80(\mathrm{~m}, 1 \mathrm{H}) ; 3.96(\mathrm{~s}, 3 \mathrm{H}) ; 4.38(\mathrm{~d}, J=8.53 \mathrm{~Hz}, 1 \mathrm{H}) ; 4.66(\mathrm{~d}, J=10.54 \mathrm{~Hz}, 1 \mathrm{H}) ; 4.79(\mathrm{~d}, J=8.28 \mathrm{~Hz}$, $1 \mathrm{H}) ; 5.16(\mathrm{~d}, J=8.53 \mathrm{~Hz}, 1 \mathrm{H}) ; 6.92-7.03(\mathrm{~m}, 4 \mathrm{H}) 7.12-7.32(\mathrm{~m}, 13 \mathrm{H}), 7.33-7.44(\mathrm{~m}, 6 \mathrm{H}), 7.45-7.55$ $(\mathrm{m}, 4 \mathrm{H}), 7.75(\mathrm{~s}, 1 \mathrm{H}) ; 7.89(\mathrm{~d}, J=7.78 \mathrm{~Hz}, 2 \mathrm{H}) .{ }^{13} \mathrm{C}-\mathrm{NMR}\left(100 \mathrm{MHz}, \mathrm{CDCl}_{3}\right) \delta \mathrm{ppm}: 48.6(\mathrm{CH}) ; 49.3$ $(\mathrm{CH}) ; 49.6(\mathrm{CH}) ; 52.8(\mathrm{CH}) ; 53.7\left(\mathrm{CH}_{3}\right) ; 59.2(\mathrm{CH}) ; 60.7(\mathrm{CH}) ; 80.2(\mathrm{C}) ; 110.8(\mathrm{C}) 119.2(\mathrm{CH}) ; 119.8$ $(\mathrm{CH}) ; 120.1(\mathrm{C}) ; 125.3(\mathrm{C}) ; 126.6(\mathrm{CH}) ; 126.8(\mathrm{CH}) ; 127.0(\mathrm{CH}) ; 127.4(\mathrm{CH}) ; 127.9(\mathrm{CH}) ; 128.0(\mathrm{CH})$; $128.2(\mathrm{CH}) ; 128.4(\mathrm{CH}) ; 128.6(\mathrm{CH}) ; 128.7(\mathrm{CH}) ; 128.9(\mathrm{C}) ; 129.1(\mathrm{CH}) ; 129.2(\mathrm{CH}) ; 129.3(\mathrm{CH})$; $129.4(\mathrm{CH}) ; 129.5(\mathrm{CH}) ; 129.6(\mathrm{C}) ; 130.0(\mathrm{CH}) ; 132.2(\mathrm{C}) ; 132.5(\mathrm{C}) ; 134.5(\mathrm{C}) ; 135.5(\mathrm{C}), 139.5(\mathrm{C})$; 139.6 (C); 150.4 (C); 152.7 (C); 169.9 (C); 172.1 (C); 173.3 (C); 175.1 (C). MS (EI, 70 eV) m/z (\%): 963( $\left.\mathrm{M}^{+}\right), 756$ (31), 523 (10), 298 (18), 273 (16), 233 (100), 207 (30). Elemental Analyses calcd. for $\mathrm{C}_{55} \mathrm{H}_{39} \mathrm{Cl}_{2} \mathrm{~N}_{7} \mathrm{O}_{6} .4 \mathrm{H}_{2} \mathrm{O}: \mathrm{C}: 63.71, \mathrm{H}: 4.57, \mathrm{~N}: 9.46$. Found: C: 63.74, H: 4.42, N: 9.37.

Methyl 7,9-bis(1,3-diphenyl-1H-pyrazol-4-yl)-1,3,4,6-tetraoxo-2,5-di-p-tolyldodecahydro-1H-dipyrrolo[3,4-a:3',4'-f]pyrrolizine-3b-carboxylate (4h). White Solid. Yield: 93\%; m.p.: 204-206 ${ }^{\circ} \mathrm{C}$. IR (KBr): v 3574, 2954, 1747, 1711, $1599 \mathrm{~cm}^{-1}$. ${ }^{1} \mathrm{H}-\mathrm{NMR}\left(400 \mathrm{MHz}, \mathrm{CDCl}_{3}\right) \delta \mathrm{ppm}: 2.26(\mathrm{~s}, 3 \mathrm{H}), 2.50(\mathrm{~s}, 3 \mathrm{H}), 3.59$ $(\mathrm{t}, J=8.4 \mathrm{~Hz}, 1 \mathrm{H}), 3.80(\mathrm{dd}, J=10.5,8.3 \mathrm{~Hz}, 1 \mathrm{H}), 3.96(\mathrm{~s}, 3 \mathrm{H}), 4.35(\mathrm{~d}, J=8.3 \mathrm{~Hz}, 1 \mathrm{H}), 4.71(\mathrm{~d}$, $J=10.8 \mathrm{~Hz}, 1 \mathrm{H}), 4.80(\mathrm{~d}, J=8.3 \mathrm{~Hz}, 1 \mathrm{H}), 5.14(\mathrm{~d}, J=8.5 \mathrm{~Hz}, 1 \mathrm{H}), 6.84(\mathrm{~d}, J=8.3 \mathrm{~Hz}, 2 \mathrm{H}), 6.93(\mathrm{~d}$, 
$J=8.0 \mathrm{~Hz}, 2 \mathrm{H}), 7.03(\mathrm{~d}, J=8.0 \mathrm{~Hz}, 2 \mathrm{H}), 7.12-7.18(\mathrm{~m}, 2 \mathrm{H}), 7.18-7.24(\mathrm{~m}, 1 \mathrm{H}), 7.26-7.30(\mathrm{~m}, 5 \mathrm{H})$, $7.30-7.44(\mathrm{~m}, 10 \mathrm{H}), 7.44-7.50(\mathrm{~m}, 3 \mathrm{H}), 7.82(\mathrm{~s}, 1 \mathrm{H}), 7.90(\mathrm{~d}, J=7.0 \mathrm{~Hz}, 2 \mathrm{H}) .{ }^{13} \mathrm{C}-\mathrm{NMR}(100 \mathrm{MHz}$, $\left.\mathrm{CDCl}_{3}\right) \delta$ ppm: $21.1\left(\mathrm{CH}_{3}\right), 21.4\left(\mathrm{CH}_{3}\right), 48.8(\mathrm{CH}), 49.5(\mathrm{CH}), 49.6(\mathrm{CH}), 52.9(\mathrm{CH}), 53.7\left(\mathrm{OCH}_{3}\right), 59.1$ $(\mathrm{CH}), 60.7(\mathrm{CH}), 80.1(\mathrm{C}), 100.0(\mathrm{C}), 111.0(\mathrm{C}), 119.3(\mathrm{CH}), 119.8(\mathrm{CH}), 120.4(\mathrm{C}), 125.5(\mathrm{CH}), 126.0$ $(\mathrm{CH}), 126.4(\mathrm{CH}), 126.7(\mathrm{CH}), 127.2(\mathrm{CH}), 127.9(\mathrm{CH}), 128.0(\mathrm{CH}), 128.2(\mathrm{CH}), 128.3(\mathrm{CH}), 128.5$ $(\mathrm{CH}), 128.6(\mathrm{CH}), 128.7(\mathrm{CH}), 129.2(\mathrm{CH}), 129.3(\mathrm{CH}), 129.7(\mathrm{CH}), 130.4(\mathrm{CH}), 132.3(\mathrm{C}), 132.6(\mathrm{C})$, 138.6 (C), 138.7 (C), 139.6 (C), 139.7 (C), 139.8 (C), 150.5 (C), 152.7 (C), 170.2 (C), 172.6 (C), 173.8 (C), 175.4 (C), 176.2 (C). MS (EI, $70 \mathrm{eV}) \mathrm{m} / z$ (\%): 923(M ), 865 (5), 737 (95), 503 (16), 298 (35), 233 (100), 187 (45). Elemental Analyses calcd. for $\mathrm{C}_{57} \mathrm{H}_{45} \mathrm{~N}_{7} \mathrm{O}_{6} \cdot \mathrm{H}_{2} \mathrm{O}: \mathrm{C}: 72.67, \mathrm{H}: 5.03, \mathrm{~N}: 10.41$. Found: C: 72.99, H: 5.24, N: 10.50 .

Methyl 7,9-bis(1,3-diphenyl-1H-pyrazol-4-yl)-2,5-bis(4-methoxyphenyl)-1,3,4,6-tetraoxododecahydro1H-dipyrrolo[3,4-a:3',4'-f]pyrrolizine-3b-carboxylate (4i). Beige Solid. Yield: 94\%; m.p.: 239-241 ${ }^{\circ} \mathrm{C}$. IR (KBr): v 3474, 2955, 1714, $1599 \mathrm{~cm}^{-1} .{ }^{1} \mathrm{H}-\mathrm{NMR}\left(400 \mathrm{MHz}, \mathrm{CDCl}_{3}\right) \delta \mathrm{ppm:} 3.57(\mathrm{t}, J=8.4 \mathrm{~Hz}, 1 \mathrm{H})$, $3.70(\mathrm{~s}, 3 \mathrm{H}), 3.76-3.80(\mathrm{~m}, 1 \mathrm{H}), 3.92(\mathrm{~s}, 3 \mathrm{H}), 3.96(\mathrm{~s}, 3 \mathrm{H}), 4.33(\mathrm{~d}, J=8.3 \mathrm{~Hz}, 1 \mathrm{H}), 4.68(\mathrm{~d}, J=10.8 \mathrm{~Hz}$, $1 \mathrm{H}), 4.79(\mathrm{~d}, J=8.3 \mathrm{~Hz}, 1 \mathrm{H}), 5.14(\mathrm{~d}, J=8.3 \mathrm{~Hz}, 1 \mathrm{H}), 6.73(\mathrm{~d}, J=9.0 \mathrm{~Hz}, 2 \mathrm{H}), 6.88(\mathrm{~d}, J=8.8 \mathrm{~Hz}$, 2H), $6.95(\mathrm{~d}, J=8.8 \mathrm{~Hz}, 2 \mathrm{H}), 7.05(\mathrm{~d}, J=8.8 \mathrm{~Hz}, 2 \mathrm{H}), 7.12-7.122(\mathrm{~m}, 4 \mathrm{H}), 7.24-7.36(\mathrm{~m}, 9 \mathrm{H}), 7.38-7.44$ $(\mathrm{m}, 4 \mathrm{H}), 7.46-7.50(\mathrm{~m}, 2 \mathrm{H}), 7.81(\mathrm{~s}, 1 \mathrm{H}), 7.91(\mathrm{~d}, J=7.0 \mathrm{~Hz}, 2 \mathrm{H}) .{ }^{13} \mathrm{C}-\mathrm{NMR}\left(100 \mathrm{MHz}, \mathrm{CDCl}_{3}\right) \delta$ ppm: $48.7(\mathrm{CH}), 49.4(\mathrm{CH}), 49.5(\mathrm{CH}), 52.8(\mathrm{CH}), 53.6\left(\mathrm{OCH}_{3}\right), 55.4\left(\mathrm{OCH}_{3}\right), 55.6\left(\mathrm{OCH}_{3}\right), 59.1$ $(\mathrm{CH}), 60.6(\mathrm{CH}), 80.1(\mathrm{C}), 111.1(\mathrm{C}), 114.3(\mathrm{CH}), 115.1(\mathrm{CH}), 119.3(\mathrm{CH}), 119.8(\mathrm{CH}), 120.5(\mathrm{C})$, $123.7(\mathrm{C}), 123.8(\mathrm{C}), 126.4(\mathrm{CH}), 126.7(\mathrm{CH}), 126.9(\mathrm{CH}), 127.1(\mathrm{CH}), 127.4(\mathrm{CH}), 127.8(\mathrm{CH}), 128.0$ $(\mathrm{CH}), 128.2(\mathrm{CH}), 128.3(\mathrm{CH}), 128.4(\mathrm{CH}), 128.5(\mathrm{CH}), 128.6(\mathrm{CH}), 129.1(\mathrm{CH}), 129.2(\mathrm{CH}), 132.3$ (C), 132.6 (C), 139.6 (C), 139.7 (C), 150.5 (C), 152.7 (C), 159.3 (C), 160.1 (C), 170.2 (C), 172.7 (C), 173.9 (C), 175.6 (C), 176.3 (C). MS (EI, $70 \mathrm{eV}) \mathrm{m} / z(\%)$ : 955(M $\left.\mathrm{M}^{+}\right), 751$ (38), 521 (25), 233 (100), 203 (48). Elemental Analyses calcd. for $\mathrm{C}_{57} \mathrm{H}_{45} \mathrm{~N}_{7} \mathrm{O}_{8} .2 \mathrm{H}_{2} \mathrm{O}$ : C: 69.01, H: 4.98, N: 9.88. Found: C: 69.42, H: 5.18, N: 9.84 .

Methyl 2,5-bis(benzo[d][1,3]dioxol-5-ylmethyl)-7,9-bis(1,3-diphenyl-1H-pyrazol-4-yl)-1,3,4,6-tetraoxododecahydro-1H-dipyrrolo[3,4-a:3',4'-f] pyrrolizine-3b-carboxylate (4j). White Solid. Yield: 75\%; m.p.: $241-243{ }^{\circ} \mathrm{C}$. IR (KBr): v 3647, 3062, 1744, 1700, 1599, $1503 \mathrm{~cm}^{-1} .{ }^{1} \mathrm{H}-\mathrm{NMR}\left(400 \mathrm{MHz}, \mathrm{CDCl}_{3}\right)$ $\delta$ ppm: 3.34 (t, $J=8.3 \mathrm{~Hz}, 1 \mathrm{H}), 3.50(\mathrm{t}, J=9.3 \mathrm{~Hz}, 1 \mathrm{H}), 3.92(\mathrm{~s}, 3 \mathrm{H}), 4.20(\mathrm{~d}, J=13.8 \mathrm{~Hz}, 1 \mathrm{H})$, $4.24-4.31(\mathrm{~m}, 2 \mathrm{H}), 4.39(\mathrm{dd}, J=13.8,5.0 \mathrm{~Hz}, 2 \mathrm{H}), 4.49-4.54(\mathrm{~m}, 1 \mathrm{H}), 4.62(\mathrm{~d}, J=8.3 \mathrm{~Hz}, 1 \mathrm{H}), 4.75$ $(\mathrm{d}, J=8.5 \mathrm{~Hz}, 1 \mathrm{H}), 5.75-5.85(\mathrm{~m}, 2 \mathrm{H}), 5.95(\mathrm{~s}, 2 \mathrm{H}), 6.34(\mathrm{~d}, J=7.8 \mathrm{~Hz}, 1 \mathrm{H}), 6.65-6.70(\mathrm{~m}, 1 \mathrm{H}), 6.74$ $(\mathrm{d}, J=8.0 \mathrm{~Hz}, 1 \mathrm{H}), 6.85-6.92(\mathrm{~m}, 3 \mathrm{H}), 6.98(\mathrm{~d}, J=7.3 \mathrm{~Hz}, 2 \mathrm{H}), 7.04-7.10(\mathrm{~m}, 3 \mathrm{H}), 7.13-7.24(\mathrm{~m}$, $5 \mathrm{H}), 7.27-7.36(\mathrm{~m}, 5 \mathrm{H}), 7.38-7.50(\mathrm{~m}, 5 \mathrm{H}), 7.86(\mathrm{~d}, J=7.3 \mathrm{~Hz}, 2 \mathrm{H}) .{ }^{13} \mathrm{C}-\mathrm{NMR}\left(100 \mathrm{MHz}, \mathrm{CDCl}_{3}\right) \delta$ ppm: $42.1\left(\mathrm{CH}_{2}\right), 42.9\left(\mathrm{CH}_{2}\right), 48.2(\mathrm{CH}), 48.3(\mathrm{CH}), 50.0(\mathrm{CH}), 52.0(\mathrm{CH}), 53.6\left(\mathrm{OCH}_{3}\right), 58.6(\mathrm{CH})$, $60.0(\mathrm{CH}), 80.3(\mathrm{C}), 100.9\left(\mathrm{CH}_{2}\right), 101.2\left(\mathrm{CH}_{2}\right), 108.1(\mathrm{CH}), 108.4(\mathrm{CH}), 109.0(\mathrm{CH}), 109.5(\mathrm{CH})$, $111.0(\mathrm{C}), 119.1(\mathrm{C}), 119.3(\mathrm{CH}), 120.0(\mathrm{CH}), 122.2(\mathrm{CH}), 122.7(\mathrm{CH}), 126.1(\mathrm{CH}), 126.6(\mathrm{CH}), 126.7$ $(\mathrm{CH}), 127.6(\mathrm{CH}), 127.8(\mathrm{CH}), 127.9(\mathrm{CH}), 128.2(\mathrm{C}), 128.3(\mathrm{CH}), 128.4(\mathrm{CH}), 128.5(\mathrm{CH}), 128.6$ $(\mathrm{CH}), 128.9(\mathrm{CH}), 129.2(\mathrm{CH}), 129.4(\mathrm{C}), 132.4(\mathrm{C}), 132.6(\mathrm{C}), 139.5(\mathrm{C}), 139.6(\mathrm{C}), 147.1(\mathrm{C}), 147.5$ (C), 147.7 (C), 147.8 (C), 150.1 (C), 152.2 (C), 170.2 (C), 173.0 (C), 174.7 (C), 176.4 (C), 176.6 (C). MS 


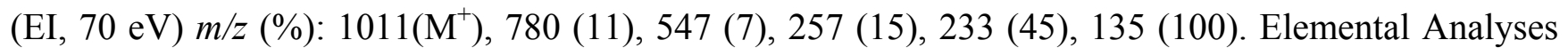
calcd. for $\mathrm{C}_{59} \mathrm{H}_{45} \mathrm{~N}_{7} \mathrm{O}_{10} .2 \mathrm{H}_{2} \mathrm{O}: \mathrm{C}: 67.61, \mathrm{H}: 4.71, \mathrm{~N}: 9.36$. Found: C: 67.84, H: 4.39, N: 9.21.

\subsection{Synthesis and Characterization Data for Pyrazolylpyrrolo[3,4-c]Pyrroles 5a-f and $\mathbf{6 a}-\mathbf{f}$.}

General Synthetic Procedure

To a $25.0 \mathrm{~mL}$ round bottom flask equipped with a magnetic stirring bar and a reflux condenser were added pyrazole-4-carboxaldehyde $\mathbf{1 a}-\mathbf{b}(0.2 \mathrm{mmol}), N$-substituted-maleimide $\mathbf{2 a}-\mathbf{e}(0.2 \mathrm{mmol})$, $N$-benzyl glycine ethyl ester $\mathbf{3 b}(0.22 \mathrm{mmol})$ and toluene $(8 \mathrm{~mL})$. The mixture was heated under reflux until TLC showed the absence of the starting materials $(6-10 \mathrm{~h})$. After the reaction mixture was cooled down to room temperature, the solvent was removed under reduced pressure and the resulting crude product was purified by column chromatography on silica gel, using a mixture of dichloromethane/hexane (7:3) as eluent. In all cases the minor diastereomers were characterized by ${ }^{1} \mathrm{H}-\mathrm{NMR}$ spectroscopy after purification.

Ethyl 2-benzyl-3-(3-methyl-1-phenyl-1H-pyrazol-4-yl)-4,6-dioxo-5-phenyloctahydropyrrolo[3,4-c]pyrrole-1-carboxylate. White Solid. Yield: 95\%; m.p.: 172-174 ${ }^{\circ} \mathrm{C}$. IR (KBr): v 1717, 1598, $1502 \mathrm{~cm}^{-1}$. Minor diastereomer 5a. ${ }^{1} \mathrm{H}-\mathrm{NMR}\left(400 \mathrm{MHz}, \mathrm{CDCl}_{3}\right) \delta \mathrm{ppm}: 1.26(\mathrm{t}, J=7.3 \mathrm{~Hz}, 3 \mathrm{H}), 2.38(\mathrm{~s}, 3 \mathrm{H}), 3.34$ $(\mathrm{d}, J=13.8 \mathrm{~Hz}, 1 \mathrm{H}), 3.57$ (dd, $J=9.7,5.4 \mathrm{~Hz}, 1 \mathrm{H}), 3.86(\mathrm{~d}, J=4.8 \mathrm{~Hz}, 1 \mathrm{H}), 3.89$ (d, $J=9.3 \mathrm{~Hz}, 1 \mathrm{H}), 4.20$ (q, $J=7.3 \mathrm{~Hz}, 2 \mathrm{H}), 4.24(\mathrm{~d}, J=9.0 \mathrm{~Hz}, 1 \mathrm{H}), 4.79(\mathrm{~d}, J=5.5 \mathrm{~Hz}, 1 \mathrm{H}), 7.22-7.28(\mathrm{~m}, 4 \mathrm{H}), 7.29-7.34$ $(\mathrm{m}, 4 \mathrm{H}), 7.40-7.53(\mathrm{~m}, 5 \mathrm{H}), 7.66(\mathrm{~d}, J=7.5 \mathrm{~Hz}, 2 \mathrm{H}), 7.92(\mathrm{~s}, 1 \mathrm{H})$. Major diastereomer $6 \mathbf{a}^{1} \mathrm{H}-\mathrm{NMR}$ $\left(400 \mathrm{MHz}, \mathrm{CDCl}_{3}\right) \delta \mathrm{ppm}: 1.32(\mathrm{t}, J=7.2 \mathrm{~Hz}, 3 \mathrm{H}), 2.45(\mathrm{~s}, 3 \mathrm{H}), 3.53(\mathrm{~d}, J=8.0 \mathrm{~Hz}, 1 \mathrm{H}), 3.65(\mathrm{~d}$, $J=14.0 \mathrm{~Hz}, 1 \mathrm{H}), 3.80-3.87(\mathrm{~m}, 1 \mathrm{H}), 4.05(\mathrm{~d}, J=14.1 \mathrm{~Hz}, 1 \mathrm{H}), 4.17-4.34$ (m, 2H), 4.39 (s, $1 \mathrm{H}), 4.94(\mathrm{~d}$, $J=9.5 \mathrm{~Hz}, 1 \mathrm{H}), 7.13(\mathrm{~d}, J=6.8 \mathrm{~Hz}, 2 \mathrm{H}), 7.19-7.30(\mathrm{~m}, 4 \mathrm{H}), 7.31-7.42(\mathrm{~m}, 7 \mathrm{H}), 7.52(\mathrm{~d}, J=7.8 \mathrm{~Hz}$, 2H), $7.72(\mathrm{~s}, 1 \mathrm{H}) .{ }^{13} \mathrm{C}-\mathrm{NMR}\left(100 \mathrm{MHz}, \mathrm{CDCl}_{3}\right) \delta \mathrm{ppm}: 12.4\left(\mathrm{CH}_{3}\right), 14.2\left(\mathrm{CH}_{3}\right), 48.6(\mathrm{CH}), 48.7(\mathrm{CH})$, $52.1\left(\mathrm{CH}_{2}\right), 59.8(\mathrm{CH}), 60.9\left(\mathrm{CH}_{2}\right), 62.9(\mathrm{CH}), 118.5(\mathrm{CH}), 125.5(\mathrm{CH}), 126.0(\mathrm{CH}), 127.3(\mathrm{CH}), 127.7$ $(\mathrm{CH}), 128.2(\mathrm{CH}), 128.3(\mathrm{CH}), 128.6(\mathrm{CH}), 129.0(\mathrm{CH}), 129.2(\mathrm{CH}), 130.3(\mathrm{C}), 131.6(\mathrm{C}), 137.6(\mathrm{C})$, 139.8 (C), 149.8 (C), 170.9 (C), 174.3 (C), 175.6 (C). MS (EI, $70 \mathrm{eV}) \mathrm{m} / z$ (\%): $534\left(\mathrm{M}^{+}, 15\right), 461$ (65), 91 (100). Elemental Analyses calcd. for $\mathrm{C}_{32} \mathrm{H}_{30} \mathrm{~N}_{4} \mathrm{O}_{4} \cdot 2 \mathrm{H}_{2} \mathrm{O}: \mathrm{C}: 67.35, \mathrm{H}: 6.01, \mathrm{~N}: 9.82$. Found: C: 67.45, H: 6.13, N: 9.66.

Ethyl 2-benzyl-5-(4-chlorophenyl)-3-(3-methyl-1-phenyl-1H-pyrazol-4-yl)-4,6-dioxooctahydropyrrolo[3,4-c]pyrrole-1-carboxylate. Yellow Solid. Yield: 82\%; m.p.: $69-71{ }^{\circ} \mathrm{C}$. IR: $v 1782,1738,1598 \mathrm{~cm}^{-1}$. Minor diastereomer 5b. ${ }^{1} \mathrm{H}-\mathrm{NMR}\left(400 \mathrm{MHz}, \mathrm{CDCl}_{3}\right) \delta \mathrm{ppm}: 1.24(\mathrm{t}, J=7.3 \mathrm{~Hz}, 3 \mathrm{H}), 2.36(\mathrm{~s}, 3 \mathrm{H}), 3.31$ $(\mathrm{d}, \mathrm{J}=13.8 \mathrm{~Hz}, 1 \mathrm{H}), 3.54(\mathrm{dd}, J=9.5,5.5 \mathrm{~Hz}, 1 \mathrm{H}), 3.77-3.85(\mathrm{~m}, 3 \mathrm{H}), 4.22-4.30(\mathrm{~m}, 2 \mathrm{H}), 4.75(\mathrm{~d}$, $J=5.3 \mathrm{~Hz}, 1 \mathrm{H}), 7.06(\mathrm{~d}, J=8.3 \mathrm{~Hz}, 2 \mathrm{H}), 7.18-7.23(\mathrm{~m}, 2 \mathrm{H}), 7.29-7.34(\mathrm{~m}, 3 \mathrm{H}), 7.37$ (t, $J=7.8 \mathrm{~Hz}$, 2H), $7.44(\mathrm{~d}, J=8.5 \mathrm{~Hz}, 1 \mathrm{H}), 7.49$ (d, $J=8.0 \mathrm{~Hz}, 2 \mathrm{H}), 7.67$ (d, $J=7.5 \mathrm{~Hz}, 2 \mathrm{H}), 7.91$ (s, 1H). Major diastereomer 6b. ${ }^{1} \mathrm{H}-\mathrm{NMR}\left(400 \mathrm{MHz}, \mathrm{CDCl}_{3}\right) \delta \mathrm{ppm}: 1.30(\mathrm{t}, J=7.2 \mathrm{~Hz}, 3 \mathrm{H}), 2.42(\mathrm{~s}, 3 \mathrm{H}), 3.50(\mathrm{~d}$, $J=7.8 \mathrm{~Hz}, 1 \mathrm{H}), 3.63(\mathrm{~d}, J=14.3 \mathrm{~Hz}, 1 \mathrm{H}), 3.78-3.81(\mathrm{~m}, 1 \mathrm{H}), 4.02(\mathrm{~d}, J=14.3 \mathrm{~Hz}, 1 \mathrm{H}), 4.15-4.22(\mathrm{~m}$, 2H), $4.35(\mathrm{~s}, 1 \mathrm{H}), 4.91(\mathrm{~d}, J=9.5 \mathrm{~Hz}, 1 \mathrm{H}), 7.06(\mathrm{~d}, J=8.3 \mathrm{~Hz}, 2 \mathrm{H}), 7.18-7.23(\mathrm{~m}, 3 \mathrm{H}), 7.29-7.34(\mathrm{~m}$, $3 \mathrm{H}), 7.37$ (t, $J=7.8 \mathrm{~Hz}, 2 \mathrm{H}), 7.44$ (d, $J=8.5 \mathrm{~Hz}, 1 \mathrm{H}), 7.49$ (d, $J=8.0 \mathrm{~Hz}, 2 \mathrm{H}), 7.63-7.69$ (m, 2H). ${ }^{13} \mathrm{C}-\mathrm{NMR}\left(100 \mathrm{MHz}, \mathrm{CDCl}_{3}\right) \delta$ ppm: $12.4\left(\mathrm{CH}_{3}\right), 14.2\left(\mathrm{CH}_{3}\right), 46.4(\mathrm{CH}), 48.7(\mathrm{CH}), 52.1\left(\mathrm{CH}_{2}\right), 59.8$ 
$(\mathrm{CH}), 60.9\left(\mathrm{CH}_{2}\right), 62.9(\mathrm{CH}), 118.4(\mathrm{CH}), 119.6(\mathrm{C}), 126.1(\mathrm{CH}), 126.6(\mathrm{CH}), 127.3(\mathrm{CH}), 127.7(\mathrm{CH})$, $128.6(\mathrm{CH}), 129.1(\mathrm{CH}), 129.2(\mathrm{CH}), 129.3(\mathrm{CH}), 130.1(\mathrm{C}), 133.9(\mathrm{C}), 137.5(\mathrm{C}), 139.7(\mathrm{C}), 170.8$ (C), 170.8 (C), 174.1 (C), 175.3 (C). MS (EI, $70 \mathrm{eV)} \mathrm{m/z} \mathrm{( \% ):} 568\left(\mathrm{M}^{+}, 1\right), 497$ (11), 496 (10), 495 (30), 91 (100). Elemental Analyses calcd. for $\mathrm{C}_{32} \mathrm{H}_{29} \mathrm{ClN}_{4} \mathrm{O}_{5} \cdot \mathrm{H}_{2} \mathrm{O}: \mathrm{C}: 65.47, \mathrm{H}: 5.32$, N: 9.54. Found: C: 65.28, H: 5.39, N: 9.76.

Ethyl 2-benzyl-5-(4-methoxyphenyl)-3-(3-methyl-1-phenyl-1H-pyrazol-4-yl)-4,6-dioxooctahydropyrrolo[3,4-c]pyrrole-1-carboxylate. White Solid. Yield: 96\%; m.p.: 85-87 ${ }^{\circ} \mathrm{C}$. IR: v 1775, 1728, $1602 \mathrm{~cm}^{-1}$. Minor diastereomer 5c. ${ }^{1} \mathrm{H}-\mathrm{NMR}\left(400 \mathrm{MHz}, \mathrm{CDCl}_{3}\right) \delta \mathrm{ppm}: 1.26(\mathrm{t}, J=7.2 \mathrm{~Hz}, 3 \mathrm{H}), 2.38$ (s, 3H), $3.34(\mathrm{~d}, J=13.8 \mathrm{~Hz}, 1 \mathrm{H}), 3.55(\mathrm{dd}, J=9.5,5.3 \mathrm{~Hz}, 1 \mathrm{H}), 3.82-3.91(\mathrm{~m}, 5 \mathrm{H}), 4.19$ (q, $J=7.3 \mathrm{~Hz}$, $2 \mathrm{H}), 4.23(\mathrm{~d}, J=8.8 \mathrm{~Hz}, 1 \mathrm{H}), 4.78(\mathrm{~d}, J=5.5 \mathrm{~Hz}, 1 \mathrm{H}), 7.00(\mathrm{~d}, J=9.0,2 \mathrm{H}), 7.21-7.26(\mathrm{~m}, 5 \mathrm{H}), 7.27-7.34$ $(\mathrm{m}, 3 \mathrm{H}), 7.45(\mathrm{t}, J=8.5,7.3 \mathrm{~Hz}, 2 \mathrm{H}), 7.66(\mathrm{dd}, J=8.7,1.1 \mathrm{~Hz}, 2 \mathrm{H}), 7.91(\mathrm{~s}, 1 \mathrm{H})$. Major diastereomer 6c. ${ }^{1} \mathrm{H}-\mathrm{NMR}\left(400 \mathrm{MHz}, \mathrm{CDCl}_{3}\right) \delta \mathrm{ppm}: 1.32(\mathrm{t}, J=7.2 \mathrm{~Hz}, 3 \mathrm{H}), 2.44(\mathrm{~s}, 3 \mathrm{H}), 3.51(\mathrm{~d}, J=7.8 \mathrm{~Hz}, 1 \mathrm{H})$, $3.65(\mathrm{~d}, J=14.3 \mathrm{~Hz}, 1 \mathrm{H}), 3.76(\mathrm{~s}, 3 \mathrm{H}), 4.04(\mathrm{~d}, J=14.1 \mathrm{~Hz}, 1 \mathrm{H}), 4.16-4.32(\mathrm{~m}, 3 \mathrm{H}), 4.38(\mathrm{~s}, 1 \mathrm{H}), 4.92$ $(\mathrm{d}, J=9.5 \mathrm{~Hz}, 1 \mathrm{H}), 6.84(\mathrm{~d}, J=9.0 \mathrm{~Hz}, 2 \mathrm{H}), 7.04(\mathrm{~d}, J=8.8 \mathrm{~Hz}, 2 \mathrm{H}), 7.21-7.27(\mathrm{~m}, 3 \mathrm{H}), 7.30-7.35(\mathrm{~m}$, $3 \mathrm{H}), 7.36-7.41(\mathrm{~m}, 2 \mathrm{H}), 7.53(\mathrm{~d}, J=7.8 \mathrm{~Hz}, 2 \mathrm{H}), 7.70(\mathrm{~s}, 1 \mathrm{H}) .{ }^{13} \mathrm{C}-\mathrm{NMR}\left(100 \mathrm{MHz}, \mathrm{CDCl}_{3}\right) \delta \mathrm{ppm}$ : $12.4\left(\mathrm{CH}_{3}\right), 14.2\left(\mathrm{CH}_{3}\right), 46.4(\mathrm{CH}), 48.6(\mathrm{CH}), 52.0\left(\mathrm{CH}_{2}\right), 55.3\left(\mathrm{OCH}_{3}\right), 59.8(\mathrm{CH}), 60.9\left(\mathrm{CH}_{2}\right), 62.9$ $(\mathrm{CH}), 114.3(\mathrm{CH}), 118.5(\mathrm{CH}), 119.8(\mathrm{C}), 124.3(\mathrm{C}), 126(\mathrm{CH}), 126.8(\mathrm{CH}), 127.3(\mathrm{CH}), 127.7(\mathrm{CH})$, $128.2(\mathrm{CH}), 128.6(\mathrm{CH}), 129.2(\mathrm{CH}), 137.7(\mathrm{C}), 139.8(\mathrm{C}), 149.8(\mathrm{C}), 159.1(\mathrm{C}), 171.0(\mathrm{C}), 174.5(\mathrm{C})$, 175.8 (C). MS (EI, $70 \mathrm{eV}) \mathrm{m} / z$ (\%): $564\left(\mathrm{M}^{+}, 6\right), 493$ (14), 492 (71), 491 (99), 473 (37), 270 (33), 91 (100). Elemental Analyses calcd. for $\mathrm{C}_{33} \mathrm{H}_{32} \mathrm{~N}_{4} \mathrm{O}_{5} .2 \mathrm{H}_{2} \mathrm{O}: \mathrm{C}: 65.99, \mathrm{H}: 6.04, \mathrm{~N}: 9.33$. Found: C: 66.12, H: 6.24, N: 9.02 .

Ethyl 2-benzyl-3-(1,3-diphenyl-1H-pyrazol-4-yl)-4,6-dioxo-5-phenyloctahydropyrrolo[3,4-c]pyrrole-1carboxylate. White Solid. Yield: 75\%; m.p.: 181-183 ${ }^{\circ} \mathrm{C}$. IR (KBr): v 1721, 1597, $1498 \mathrm{~cm}^{-1}$. Minor diastereomer 5d. ${ }^{1} \mathrm{H}-\mathrm{NMR}\left(400 \mathrm{MHz}, \mathrm{CDCl}_{3}\right) \delta \mathrm{ppm}: 1.27(\mathrm{t}, J=7.1 \mathrm{~Hz}, 3 \mathrm{H}), 3.38(\mathrm{~d}, J=13.6 \mathrm{~Hz}$, $1 \mathrm{H}), 3.72(\mathrm{dd}, J=9.7,5.6 \mathrm{~Hz}, 1 \mathrm{H}), 3.83(\mathrm{~d}, J=9.0 \mathrm{~Hz}, 1 \mathrm{H}), 3.89-3.93(\mathrm{~m}, 1 \mathrm{H}), 4.28-4.32(\mathrm{~m}, 3 \mathrm{H})$, $5.05(\mathrm{~d}, J=5.5 \mathrm{~Hz}, 1 \mathrm{H}), 7.14-7.18(\mathrm{~m}, 2 \mathrm{H}), 7.32-7.35(\mathrm{~m}, 5 \mathrm{H}), 7.40-7.43(\mathrm{~m}, 4 \mathrm{H}), 7.50-7.53(\mathrm{~m}, 5 \mathrm{H})$, $7.82-7.85(\mathrm{~m}, 2 \mathrm{H}), 7.97-8.02(\mathrm{~m}, 2 \mathrm{H}), 8.15(\mathrm{~s}, 1 \mathrm{H})$. Major diastereomer $6 \mathbf{6 d} .{ }^{1} \mathrm{H}-\mathrm{NMR}(400 \mathrm{MHz}$, $\left.\mathrm{CDCl}_{3}\right) \delta$ ppm: $1.32(\mathrm{t}, J=7.2 \mathrm{~Hz}, 3 \mathrm{H}), 3.58(\mathrm{~d}, J=7.8 \mathrm{~Hz}, 1 \mathrm{H}), 3.67(\mathrm{~d}, J=14.3 \mathrm{~Hz}, 1 \mathrm{H}), 3.92-3.98$ $(\mathrm{m}, 1 \mathrm{H}), 4.06(\mathrm{~d}, J=14.1 \mathrm{~Hz}, 1 \mathrm{H}), 4.21-4.29(\mathrm{~m}, 2 \mathrm{H}), 4.45(\mathrm{~s}, 1 \mathrm{H}), 5.11(\mathrm{~d}, J=9.8 \mathrm{~Hz}, 1 \mathrm{H}), 7.22-7.26$ $(\mathrm{m}, 3 \mathrm{H}), 7.30(\mathrm{dd}, J=7.4,1.9 \mathrm{~Hz}, 2 \mathrm{H}), 7.32-7.35(\mathrm{~m}, 1 \mathrm{H}), 7.36-7.40(\mathrm{~m}, 4 \mathrm{H}), 7.43-7.48(\mathrm{~m}, 2 \mathrm{H})$, 7.49-7.52 (m, 2H), 7.55-7.60 (m, 2H), $7.65(\mathrm{~d}, J=7.5 \mathrm{~Hz}, 2 \mathrm{H}), 7.86-7.90(\mathrm{~m}, 2 \mathrm{H}), 7.92(\mathrm{~s}, 1 \mathrm{H})$. ${ }^{13} \mathrm{C}-\mathrm{NMR}\left(100 \mathrm{MHz}, \mathrm{CDCl}_{3}\right) \delta \mathrm{ppm}: 14.3\left(\mathrm{CH}_{3}\right), 49.0(\mathrm{CH}), 49.5(\mathrm{CH}), 52.3\left(\mathrm{CH}_{2}\right), 60.2(\mathrm{CH}), 61.1$ $\left(\mathrm{CH}_{2}\right), 63.1(\mathrm{CH}), 118.9(\mathrm{CH}), 119.0(\mathrm{CH}), 119.1(\mathrm{C}), 125.6(\mathrm{CH}), 126.1(\mathrm{CH}), 126.6(\mathrm{CH}), 126,7$ $(\mathrm{CH}), 127.4(\mathrm{CH}), 128.4(\mathrm{CH}), 128.5(\mathrm{CH}), 128.6(\mathrm{CH}), 128.7(\mathrm{CH}), 129.2(\mathrm{CH}), 129.4(\mathrm{CH}), 131.8$ (C), 133.1 (C), 137.8 (C), 139.9 (C), 153.3 (C), 171.0 (C), 174.6 (C), 175.7 (C). MS (EI, 70 eV) m/z (\%): $596\left(\mathrm{M}^{+}, 4\right), 523$ (100), 505 (32). Elemental Analyses calcd. for $\mathrm{C}_{37} \mathrm{H}_{32} \mathrm{~N}_{4} \mathrm{O}_{4} \cdot \mathrm{H}_{2} \mathrm{O}: \mathrm{C}: 72.30, \mathrm{H}$ : 5.58, N: 9.11. Found: C: 72.68, H: 5.28, N: 9.05.

Ethyl 2-benzyl-5-(4-chlorophenyl)-3-(1,3-diphenyl-1H-pyrazol-4-yl)-4,6-dioxooctahydropyrrolo[3,4c]pyrrole-1-carboxylate. White Solid. Yield: 72\%; m.p.: 108-110 ${ }^{\circ} \mathrm{C}$. IR (KBr): v 1719, 1599, $1496 \mathrm{~cm}^{-1}$. 
Minor diastereomer 5e. ${ }^{1} \mathrm{H}-\mathrm{NMR}\left(400 \mathrm{MHz}, \mathrm{CDCl}_{3}\right) \delta \mathrm{ppm}: 1.23(\mathrm{t}, J=7.0 \mathrm{~Hz}, 3 \mathrm{H}), 3.33(\mathrm{~d}, J=13.6 \mathrm{~Hz}$, $1 \mathrm{H}), 3.68(\mathrm{dd}, J=9.7,5.6 \mathrm{~Hz}, 1 \mathrm{H}), 3.75-3.83(\mathrm{~m}, 1 \mathrm{H}), 3.86-3.89(\mathrm{~m}, 1 \mathrm{H}), 3.92(\mathrm{~d}, J=8.0 \mathrm{~Hz}, 1 \mathrm{H})$, 4.15-4.24 (m, 2H) $4.97(\mathrm{~d}, J=5.5 \mathrm{~Hz}, 1 \mathrm{H}), 7.09-7.13(\mathrm{~m}, 2 \mathrm{H}), 7.22-7.26(\mathrm{~m}, 3 \mathrm{H}), 7.32-7.36(\mathrm{~m}, 2 \mathrm{H})$, 7.41 (br. s., $3 \mathrm{H}$ ), 7.50 (d, $J=3.3 \mathrm{~Hz}, 2 \mathrm{H}), 7.52-7.55$ (m, 3H), 7.78 (br. s., 3H), 7.94 (d, $J=6.8 \mathrm{~Hz}, 1 \mathrm{H}$ ), $8.10(\mathrm{~s}, 1 \mathrm{H})$. Major diastereomer 6e. ${ }^{1} \mathrm{H}-\mathrm{NMR}\left(400 \mathrm{MHz}, \mathrm{CDCl}_{3}\right) \delta \mathrm{ppm}: 1.28(\mathrm{t}, J=7.2 \mathrm{~Hz}, 3 \mathrm{H}), 3.53$ $(\mathrm{d}, J=7.8 \mathrm{~Hz}, 1 \mathrm{H}), 3.62(\mathrm{~d}, J=14.1 \mathrm{~Hz}, 1 \mathrm{H}), 3.88-3.95(\mathrm{~m}, 1 \mathrm{H}), 4.00(\mathrm{~d}, J=14.3 \mathrm{~Hz}, 1 \mathrm{H}), 4.12-4.21$ $(\mathrm{m}, 2 \mathrm{H}), 4.39(\mathrm{~s}, 1 \mathrm{H}), 5.05(\mathrm{~d}, J=9.8 \mathrm{~Hz}, 1 \mathrm{H}), 7.15-7.20(\mathrm{~m}, 3 \mathrm{H}), 7.20-7.26(\mathrm{~m}, 2 \mathrm{H}), 7.29-7.32(\mathrm{~m}$, 4H), 7.39-7.48 (m, 4H), 7.49-7.55 (m, 2H), $7.58(\mathrm{~d}, J=7.8 \mathrm{~Hz}, 2 \mathrm{H}), 7.76-7.85(\mathrm{~m}, 3 \mathrm{H}) .{ }^{13} \mathrm{C}-\mathrm{NMR}$ $\left(100 \mathrm{MHz}, \mathrm{CDCl}_{3}\right) \delta$ ppm: $14.2\left(\mathrm{CH}_{3}\right), 48.9(\mathrm{CH}), 49.4(\mathrm{CH}), 52.2\left(\mathrm{CH}_{2}\right), 60.1(\mathrm{CH}), 61.0\left(\mathrm{CH}_{2}\right), 63.0$ $(\mathrm{CH}), 118.8(\mathrm{CH}), 125.8(\mathrm{C}), 126.7(\mathrm{CH}), 127.4(\mathrm{CH}), 127.8(\mathrm{CH}), 128.3(\mathrm{CH}), 128.4(\mathrm{CH}), 128.5$ $(\mathrm{CH}), 128.5(\mathrm{CH}), 128.7(\mathrm{CH}), 129.1(\mathrm{CH}), 129.3(\mathrm{CH}), 129.4(\mathrm{CH}), 130.2(\mathrm{C}), 132.9(\mathrm{C}), 134.1(\mathrm{C})$, 137.6 (C), 139.7 (C), 153.2 (C), 170.8 (C), 174.2 (C), 175.4 (C). MS (EI, $70 \mathrm{eV}) \mathrm{m} / z$ (\%): $630\left(\mathrm{M}^{+}, 2\right)$, 557 (49), 554 (43), 553 (100), 535 (45). Elemental Analyses calcd. for $\mathrm{C}_{37} \mathrm{H}_{31} \mathrm{ClN}_{4} \mathrm{O}_{4}: \mathrm{C}: 70.41, \mathrm{H}$ : 4.95, N: 8.88. Found: C: 70.72, H: 5.04, N: 8.54.

Ethyl 2-benzyl-3-(1,3-diphenyl-1H-pyrazol-4-yl)-5-(4-methoxyphenyl)-4,6-dioxooctahydropyrrolo[3,4c]pyrrole-1-carboxylate. Yellow Solid. Yield: 90\%; m.p.: 78-80 ${ }^{\circ} \mathrm{C}$. IR: v 1712, 1600, $1548 \mathrm{~cm}^{-1}$. Minor diastereomer 5f. ${ }^{1} \mathrm{H}-\mathrm{NMR}\left(400 \mathrm{MHz}, \mathrm{CDCl}_{3}\right) \delta \mathrm{ppm}: 1.26(\mathrm{t}, J=7.0 \mathrm{~Hz}, 3 \mathrm{H}), 3.37(\mathrm{~d}, J=13.6 \mathrm{~Hz}$, $1 \mathrm{H}), 3.71(\mathrm{~d}, J=5.5 \mathrm{~Hz}, 1 \mathrm{H}), 3.82-3.86(\mathrm{~m}, 4 \mathrm{H}), 3.88-3.91(\mathrm{~m}, 1 \mathrm{H}), 4.21-4.31(\mathrm{~m}, 3 \mathrm{H}), 5.04(\mathrm{~d}$, $J=5.5 \mathrm{~Hz}, 1 \mathrm{H}), 7.01(\mathrm{~d}, J=9.0,2 \mathrm{H}), 7.27-7.32(\mathrm{~m}, 6 \mathrm{H}), 7.49-7.56(\mathrm{~m}, 7 \mathrm{H}), 7.84(\mathrm{~d}, J=7.8,2 \mathrm{H}), 8.00$ $(\mathrm{d}, J=7.0,2 \mathrm{H}), 8.15(\mathrm{~s}, 1 \mathrm{H})$. Major diastereomer $6 \mathbf{f} .{ }^{1} \mathrm{H}-\mathrm{NMR}\left(400 \mathrm{MHz}, \mathrm{CDCl}_{3}\right) \delta \mathrm{ppm}: 1.31(\mathrm{t}$, $J=7.2 \mathrm{~Hz}, 3 \mathrm{H}), 3.55(\mathrm{~d}, J=8.0 \mathrm{~Hz}, 1 \mathrm{H}), 3.66(\mathrm{~d}, J=14.3 \mathrm{~Hz}, 1 \mathrm{H}), 3.80$ (s, 3H), 3.93 (dd, $J=9.5 \mathrm{~Hz}$, $8.0 \mathrm{~Hz} 1 \mathrm{H}), 4.05(\mathrm{~d}, J=14.1 \mathrm{~Hz}, 1 \mathrm{H}), 4.15-4.28(\mathrm{~m}, 2 \mathrm{H}), 4.44(\mathrm{~s}, 1 \mathrm{H}), 5.10$ (d, $J=9.5 \mathrm{~Hz}, 1 \mathrm{H}), 6.89$ $(\mathrm{d}, J=9.0 \mathrm{~Hz}, 2 \mathrm{H}), 7.16(\mathrm{~d}, J=8.8 \mathrm{~Hz}, 2 \mathrm{H}), 7.21-7.27(\mathrm{~m}, 2 \mathrm{H}), 7.28-7.32(\mathrm{~m}, 2 \mathrm{H}), 7.32-7.38(\mathrm{~m}$, 2H), 7.42-7.52 (m, 3H), $7.57(\mathrm{t}, J=7.4 \mathrm{~Hz}, 2 \mathrm{H}), 7.66(\mathrm{~d}, J=7.5 \mathrm{~Hz}, 2 \mathrm{H}), 7.88(\mathrm{~d}, J=7.0 \mathrm{~Hz}, 2 \mathrm{H})$, $7.91(\mathrm{~s}, 1 \mathrm{H}) .{ }^{13} \mathrm{C}-\mathrm{NMR}\left(100 \mathrm{MHz}, \mathrm{CDCl}_{3}\right) \delta \mathrm{ppm}: 14.1\left(\mathrm{CH}_{3}\right), 48.7(\mathrm{CH}), 49.3(\mathrm{CH}), 52.1\left(\mathrm{CH}_{2}\right), 55.3$ $\left(\mathrm{OCH}_{3}\right), 60.0(\mathrm{CH}), 60.9\left(\mathrm{CH}_{2}\right), 63.0(\mathrm{CH}), 114.2(\mathrm{CH}), 118.7(\mathrm{CH}), 119.8(\mathrm{C}), 124.3(\mathrm{C}), 126.4(\mathrm{CH})$, $126.7(\mathrm{CH}), 127.2(\mathrm{CH}), 127.7(\mathrm{CH}), 128.1(\mathrm{CH}), 128.3(\mathrm{CH}), 128.4(\mathrm{CH}), 128.5(\mathrm{CH}), 129.0(\mathrm{CH})$, $129.3(\mathrm{CH}), 132.9(\mathrm{C}), 137.7$ (C), 139.7 (C), 153.1 (C), 159.1 (C), 170.8 (C), 174.6 (C), 175.8 (C). MS (EI, $70 \mathrm{eV}) \mathrm{m} / z(\%): 626\left(\mathrm{M}^{+}, 4\right), 554$ (39), 553 (100), 535 (42). Elemental analyses calcd. for $\mathrm{C}_{38} \mathrm{H}_{34} \mathrm{~N}_{4} \mathrm{O}_{5} \cdot \mathrm{H}_{2} \mathrm{O}: \mathrm{C}: 70.79, \mathrm{H}: 5.63$, N: 8.69. Found: C: 70.56, H: 5.81, N: 8.51.

\section{Conclusions}

We described here a practical synthesis of pyrazoylpyrrolizines 4 and pyrazolylpyrrolidines derivatives 5 and $\mathbf{6}$ from pyrazolyl-carboxaldehydes, glycine derivates and maleimides by a three-component catalyst free domino process involving both the formation of a 1,3-dipolar species and 1,3-cycloaddition reaction to afford the desired products in good yields and with good atom economy. This high-throughput methodology provides an easy execution, rapid access and good diastereoselectivity. When the $\mathrm{N}$-benzyl glycine ethyl ester was used two diastereomers $\mathbf{5}$ and $\mathbf{6}$ were obtained, with the diastereomers 6a-f being favored by the minor repulsive interaction between the carbonyl group on 1-C and $6-\mathrm{C}=\mathrm{O}$ carbon due to their trans configuration. 


\section{Acknowledgments}

Authors thank COLCIENCIAS, Universidad del Valle, the Spanish "Consejería de Innovación, Ciencia y Empresa, Junta de Andalucía" and "Centro de Instrumentación Científico-Técnica de la Universidad de Jaén for financial support.

\section{Author Contributions}

JQ, JG, RA, BI, AO, JC and MN designed research. All authors read and approved the final manuscript.

\section{Conflicts of Interest}

The authors declare no conflict of interest.

\section{References}

1. Sridharan, V.; Perumal, P.; Avendaño, C.; Menéndez, C. A New Three-Component Domino Synthesis of 1,4-Dihydropyridines. Tetrahedron 2007, 63, 4407-4413.

2. Ugi, I. Recent Progress in the Chemistry of Multicomponent Reactions. Pure Appl. Chem. 2001, 73, 187-191.

3. Zhu, J., Bienaymé, H., Eds. Multicomponent Reactions; Wiley-VCH: Weinheim, Germany, 2005.

4. Lieby-Muller, F.; Constantieux, T.; Rodriguez, J. Highly Efficient Access to Original Polycyclic Pyrrolopiperazine Scaffolds by a Three-Component Reaction with 1,3-Dicarbonyls. Synlett 2007, 8, 1323-1325.

5. Ruijter, E.; Scheffelaar, R.; Orru, R.V. A. Multicomponent Reaction Design in the Quest for Molecular Complexity and Diversity. Angew. Chem. Int. Ed. 2011, 50, 6234-6246.

6. Ramón, D.J.; Yus, M. Asymmetric Multicomponent Reactions (AMCRs): The New Frontier. Angew. Chem. Int. Ed. 2005, 44, 1602-1634.

7. Ayerbe, M.; Arrieta, A.; Cossío, F. Stereocontrolled Synthesis of Highly Substituted Proline Esters via [3 2] Cycloaddition between N-Metalated Azomethine Ylides and Nitroalkenes. Origins of the Metal Effect on the Stereochemical Outcome. J. Org. Chem. 1998, 63, 1795-1805.

8. Garner, P.; Kaniskan, Ü. Synthesis of Highly Functionalized Pyrrolidines via a Mild One-Pot, Three-Component 1,3-Dipolar Cycloaddition Process. J. Org. Chem. 2005, 70, 10868-10871.

9. Nájera, C.; Sansano, J. Enantioselective Synthesis of Proline Derivatives by 1,3-Dipolar Cycloadditions. Monatsh. Chem. 2011, 142, 659-680.

10. Wang, G.; Chen, Y.; Wang, S.; Gentles, R.; Sowin, T.; Kati, W.; Muchmore, S.; Giranda, V.; Stewart, K.; Sham, H.; et al. Design, Synthesis, and Structural Analysis of Influenza Neuraminidase Inhibitors Containing Pyrrolidine Cores. J. Med. Chem. 2001, 44, 1192-1201.

11. Belskaya, N.; Bakulev, V.; Deryavina, T.; Subbotina, J.; Koddes, M.; Dehaen, W.; Toppet, S.; Robeyns, K.; van Meervely, L. 3-Alkylsulfanyl-2-Arylazo-3-(pyrrolidin-1-yl)-Acrylonitriles as Masked 1,3-Dipoles. Tetrahedron 2009, 65, 7662-7672.

12. Kathiravan, S.; Ramesh, E.; Raghunathan, R. Synthesis of pyrrolo[2,3-a]pyrrolizine and pyrrolizine[2,3-a]pyrrolizine Derived from Allyl Derivatives of Baylis-Hillman Adducts through Intramolecular 1,3-Dipolar Cycloaddition. Tetrahedron Lett. 2009, 50, 2389-2391. 
13. Barker. G.; McGrath, J.; Klapars, A.; Stead, D.; Zhou, G.; Campos, K.; O’Brien, P. Enantioselective, Palladium-Catalyzed $\alpha$-Arylation of $N$-Boc Pyrrolidine: In Situ React IR Spectroscopic Monitoring, Scope, and Synthetic Applications. J. Org. Chem. 2011, 76, 5936-5953.

14. Yao, S.; Gallenkamp, D.; Wölfel, K.; Lüke, B.; Schindler, M.; Scherkenbeck, J. Synthesis and SERCA Activities of Structurally Simplified Cyclopiazonic Acid Analogues. Bioorg. Med. Chem. 2011, 19, 4669-4678.

15. Deslandes, S.; Lamoral-Theys, D.; Frongia, C.; Chassaing, S.; Bruyùre, C.; Lozach, O.; Meijer, L.; Ducommun, B.; Kiss, R.; Delfourne, D. Synthesis and Biological Evaluation of Analogs of the Marine Alkaloids Granulatimide and Isogranulatimide. Eur. J. Med. Chem. 2012, 54, 626-636.

16. Eklund, E.; Pike, R.; Scheerer, J. Synthesis of 1-Aminopyrrolizidine Alkaloid (-)-Absouline by Stereoselective Aminoconjugate Addition. Tetrahedron Lett. 2012, 53, 4644-4647.

17. Kang, T.; Cheng, Y.; He, L.; Ye, J.; Liu, Q. Facile Synthesis of Highly Functional Pyrrolizidine Derivatives from $\beta, \gamma$-Unsaturated $\alpha$-Keto Esters and Proline via a Tandem Cycloaddition. Tetrahedron Lett. 2012, 53, 2552-2555.

18. Toyooka, N.; Zhou, D.; Tezuka, Y.; Kadota, S.; Andriamaharavo, N.; Martin Garraffo, H.; Spande, T.; Daly, J. Efficient Enantio- and Diastereodivergent Synthesis of Poison-Frog Alkaloids 2510 and Trans-223B. J. Org. Chem. 2009, 74, 6784-6791.

19. Stevens, K.; Tyrell, A.; Skerratt, S.; Robertson, J. Synthesis of NP25302. Org. Lett. 2011, 13, 5964-5967.

20. Georgiou, D.; Toutountzoglou, V.; Muir, K.; Hadjipavlou-Litina, D.; Elemes, J. Synthesis of Sulfur Containing Dihydro-Pyrrolo Derivatives and Their Biological Evaluation as Antioxidants. Bioorg. Med. Chem. 2012, 20, 5103-5109.

21. Rotstein, D.; Melville, C.; Padilla, F.; Cournoyer, D.; Lee, E.; Lemoine, R.; Petersen, A.; Setti, L.; Wanner, J.; Chen, L.; et al. Novel hexahydropyrrolo[3,4-c]pyrrole CCR5 Antagonists. Bioorg. Med. Chem. 2010, 20, 3116-3119.

22. Zhang, K.; Tieke, B.; Forgie, J.; Vilela, F.; Parkinson, J.; Skabara, P. Cross-Linked Polymers Based on 2,3,5,6-Tetra-Substituted pyrrolo[3,4-c]pyrrole-1,4(2H,5H)-Dione (DPP): Synthesis, Optical and Electronic Properties. Polymer 2010, 51, 6107-6114.

23. Quiroga, J.; Portilla, J.; Abonía, R.; Insuasty, B.; Nogueras, J.; Cobo, J. Synthesis of Novel 5-Amino-1-Aroylpyrazoles. Tetrahedron Lett. 2008, 49, 5943-5945.

24. Sharma, P.; Kumar, S.; Kumar, P.; Kaushik, P.; Kaushik, D.; Dhingra, Y.; Aneja, K. Synthesis and Biological Evaluation of Some Pyrazolylpyrazolines as Anti-Inflammatory-antimicrobial Agents. Eur. J. Med. Chem. 2010, 45, 2650-2655.

25. Chauhan, A.; Sharma, P.; Kaushik, N. Pyrazole: A Versatile Moiety. Int. J. ChemTech Res. 2011, 3, 11-17.

26. Fustero, S.; Sánchez-Roselló, M.; Barrio, P.; Simón-Fuentes, A. From 2000 to Mid-2010: A Fruitful Decade for the Synthesis of Pyrazoles. Chem. Rev. 2011, 111, 6984-7034.

27. Hassan, H.; Habib, O.; Moawad, E.; El-Bana, G.; Defrawy, A. Synthesis of Some Novel Antioxidant and Anticorrosive Additives for Egyptian Gasoline Motor Oils. Lubr. Sci. 2011, 23, 119-138.

28. Quiroga, J.; Gálvez, J.; Pérez, A.; Valencia, A.; Abonía, R.; Insuasty, B. Catalyst Free Three-Component Synthesis of ( \pm )-Pyrazolylpyrrolopyrroles by 1,3-Dipolar Cycloaddition Reaction. Tetrahedron Lett. 2011, 52, 5471-5473. 
29. Quiroga, J.; Gálvez, J.; Cobo, J.; Glidewell, C. Two Methyl 3-(1H-Pyrazol-4yl) octahydropyrrolo[3,4-c]pyrrole-1-Carboxylates Form Different Hydrogen-Bonded Sheets. Acta Cryst. 2013, 69, 915-919.

30. Quiroga, J.; Portillo, S.; Pérez, A.; Gálvez, J.; Abonía, R.; Insuasty, B. An Efficient Synthesis of pyrazolo[3,4-b]pyridine-4-spiroindolinones by a Three-Component Reaction of 5-Aminopyrazoles, Isatin, and Cyclic $\beta$-Diketones. Tetrahedron Lett. 2011, 52, 2664-2666.

31. Quiroga, J.; Trilleras, J.; Pantoja, D.; Abonía, R.; Insuasty, B. Microwave-Assisted Synthesis of pyrazolo[3,4-b]pyridine-Spirocycloalkanediones by Three-Component Reaction of 5-Aminopyrazole Derivatives, Paraformaldehyde and Cyclic $\beta$-Diketones. Tetrahedron Lett. 2010, 51, 4717-4719.

32. Cui, P.; Xu, L.; Shi, Z.; Gan, L. Synthesis of Decahydropyrrolo[2,1,5-cd]indolizine through Consecutive [2+3] Cycloadditions and 6-Exo-Trig Cyclization. J. Org. Chem. 2011, 76, 4210-4212.

33. Cui, P.; Xu, L.; Cheng, H.; Gan, L. Synthesis of decahydropyrrolo[2,1,5-cd]indolizine Derivatives through $\mathrm{RuCl}_{3} / \mathrm{AgOTf}$ Induced Alkene-alkene and Alkene-arene Double Cycloisomerizations. Tetrahedron 2012, 68, 152-158.

34. Lu, Q.; Song, G.; Jasinski, J.; Keeley, A.; Zhang, W. One-Pot Double [3 + 2] Cycloaddition for Diastereoselective Synthesis of Tetracyclic Pyrrolidine Compounds. Green. Chem. 2012, 14, 3010-3012.

35. Petrovskaia, O.; Taylor, B.; Hauze, D.; Carroll, P.; Joullié, M. Investigations of the Reaction Mechanisms of 1,2-Indanediones with Amino Acids. J. Org. Chem. 2001, 66, 7666-7675.

36. Bashiardes, G.; Safir, I.; Said-Mohamed, A.; Barbot, F.; Laduranty, J. Microwave-Assisted [3 + 2] Cycloadditions of Azomethine Ylides. Org. Lett. 2003, 5, 4915-918.

37. Elboray, E.; Grigg, R.; Fishwick, C.; Kilner, C.; Sarker, M.; Aly, M.; Abbas, H. XY-ZH Compounds as Potential 1,3-Dipoles. Part 65: Atom Economic Cascade Synthesis of Highly Functionalized Pyrimidinylpyrrolidines. Tetrahedron 2011, 67, 5700-5710.

38. Quiroga, J.; Gálvez, J.; Cobo, J.; Glidewell, C. Methyl (3aRS,3cRS,6cSR,7RS,8RS,8aSR)-2, 5-bis(4-Chlorophenyl)-7,9-bis(1,3-Diphenyl-1H-Pyrazol-4-yl)-1,3,4,6-Tetraoxododecahydro-1 $H$ dipyrrolo[3,4-a:3',4'-f]pyrrolizine-3b-Carboxylate Dimethylformamide Disolvate: A Three Dimensional Hydrogen-Bonded Framework. Acta Cryst. 2012, 68, 439-442.

39. Kudryavtsev, K.; Irkha, V. Three-Component Synthesis of Polysubstituted Homoproline Analogs. Molecules 2005, 10, 755-761.

40. Frisch, M.J.; Trucks, G.W.; Schlegel, H.B.; Scuseria, G.E.; Robb, M.A.; Cheeseman, J.R.; Scalmani, G.; Barone, V.; Mennucci, B.; Petersson, G.A.; et al. Gaussian 09, Revision D.01; Gaussian, Inc.: Wallingford, CT, USA, 2009.

41. Karplus, M. Contact Electron-Spin Coupling of Nuclear Magnetic Moments. J. Chem. Phys. 1959, 30, 11-15.

42. Kalgutkar, A.; Crews, B.C.; Marnett, L.J. Design, Synthesis, and Biochemical Evaluation of $N$ Substituted Maleimides as Inhibitors of Prostaglandin Endoperoxide Synthases ${ }^{\dagger}$. J. Med. Chem. 1996, 39, 1692-1703.

Sample Availability: Samples of the compounds $4 \mathbf{a}-\mathbf{j}, \mathbf{5 a}-\mathbf{f}$ and $\mathbf{6 a}-\mathbf{f}$ are available from the authors.

(C) 2014 by the authors; licensee MDPI, Basel, Switzerland. This article is an open access article distributed under the terms and conditions of the Creative Commons Attribution license (http://creativecommons.org/licenses/by/3.0/). 